\title{
Planning a sustainable reverse logistics system: Balancing costs with environmental and social concerns
}

\author{
Tânia Rodrigues Pereira Ramos ${ }^{\mathrm{a}, *}$, Maria Isabel Gomes ${ }^{\mathrm{b}}$, Ana Paula Barbosa-Póvoa ${ }^{\mathrm{c}}$ \\ a Instituto Universitário de Lisboa (ISCTE-IUL), Business Research Unit (BRU), Av. das Forças Armadas, 1649-026 Lisboa, Portugal \\ ${ }^{\mathrm{b}}$ CMA-FCT, Universidade Nova de Lisboa, Campus da Caparica, 2829-516 Caparica, Portugal

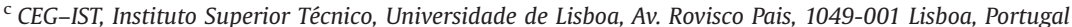

\section{A R T I C L E I N F O}

\section{Article history:}

Received 26 September 2012

Accepted 29 November 2013

Processed by B. Lev

Available online 12 December 2013

\section{Keywords:}

Sustainability

Green logistics

Social logistics

Service areas

Routing

Scheduling

\begin{abstract}
A B S T R A C T
The present work aims to support tactical and operational planning decisions of reverse logistics systems while considering economic, environmental and social objectives. In the literature, when addressing such systems economic aspects have been often used, while environmental concerns have emerged only recently. The social component is the one less studied and rarely the combination of the three concerns has been analyzed. This work considers the three objectives and was motivated by the challenge of supporting decision makers when managing a real case study of a recyclable waste collection system, where strategic decisions on the number and location of depots, vehicles and containers were taken beforehand. Tactical and operational decisions are studied involving the establishment of service areas for each depot and the definition and scheduling of collection routes for each vehicle. Such decisions should represent a compromise solution between the three objectives translating a sustainable reverse logistics plan. The problem is modeled as a multi-objective, multi-depot periodic vehicle routing problem with inter-depot routes. A mathematical formulation and a solution approach are proposed. An approximation to the Pareto front is obtained for the case study and the trade-offs between the objectives are discussed. A balanced solution is proposed.
\end{abstract}

(c) 2013 Elsevier Ltd. All rights reserved.

\section{Introduction}

Sustainability is nowadays an increasing society concern demanding for an active organizations posture. Within such context, logistics organizations play a crucial role, due to their importance in society. The design, plan and operation of sustainable logistics systems are then a challenge for the involved companies. To respond to such challenges, companies must effectively manage their logistics structures while considering economic, environmental and social objectives. Due to the complexity involved in the associated decision levels, tools that may support the decision-making process are required and represent an important defy to the academic community.

The concept of sustainability, although quite old, is commonly referred as defined in the Brundtland Report by the World Commission on Environment and Development (WCED) as "the ability to meet the needs of the present without compromising the ability of future generations to meet their own needs" [1]. To achieve such goal the three dimensions of sustainability - economic, environmental and social - need to be considered when addressing sustainable systems [2]. This is not, however, a common approach in the literature. The majority of the published works on logistics networks

\footnotetext{
* Corresponding author. Tel.: +351 217903412; fax: +351217903904.

E-mail addresses: tania.ramos@iscte.pt (T.R.P. Ramos), mirg@fct.unl.pt (M.I. Gomes), apovoa@tecnico.ulisboa.pt (A.P. Barbosa-Póvoa).
}

has looked into problems with an economic view and, only in some cases, environmental aspects have been tackled [3]. Furthermore, literature addressing the social component is scarce [4].

Some authors have investigated the environmental dimension when studying logistics decisions. This is in the case of Frota et al. [5] who developed a framework for the design and evaluation of sustainable logistics networks, where profitability and environmental impacts are balanced. Dekker et al. [6] review the contribution of Operations Research to green logistics focusing on the design, planning and control of a logistics network. Bektas and Laporte [7] introduced the pollution-routing problem, where the cost of $\mathrm{CO}_{2}$ emissions along with the operational costs of drivers and fuel consumptions are minimized when defining vehicle routes. Ubeda et al. [8] solve a vehicle routing problem with an environmental criterion minimization. Erdogan and Miller-Hooks [9] introduced the green vehicle routing problem, where an alternative fuel vehicle fleet is considered. A different way of reflecting environmental concerns in logistics decisions is to manage the returned product flow and/or integrate both forward and reverse flows in the supply chains. This topic has been intensively studied in the literature in recent years (see, for example, the works of Sheu et al. [10], Gu and Ji [11], Srivastava [12], Lee et al. [13], Salema et al. [14,15], and Qiang et al. [16]).

On the social dimension, Labuschagne et al. [17] categorize social sustainability issues into four main areas, being equity and safety within the internal human resources category, along with 
job opportunities, labor sources, diversity, discrimination, flexible working arrangements, research and development, career development, among others. Some of these aspects have been hardly explored within logistics systems. Ramos and Oliveira [18] study an organizational concern when defining service areas of a logistics system with multiple depots so as to pursuit equity. The authors state that balancing the workload (working hours) among depots is essential in problems with multiple depots, where the human resources, although part of the same organization, are fixed at each depot. A heuristic model to define depots service areas is proposed, where the minimization of the workload differences among depots is taken into account. Faulin et al. [19] address safety concerns when defining vehicle routes. These concerns are related with potential accidents in the workplace due to loading, unloading or handling activities. The authors state that avoiding workplace accidents will make logistics activities safer and healthier. Environmental concerns are also tackled, namely noise and polluting emissions. The safety and environmental concerns are translated into costs and a heuristic algorithm that optimizes the total cost is developed. Li et al. [20] address the social dimension in a truck scheduling problem for solid waste collection. A heuristic model is developed. This balances the collection routes assigned to recycling facilities in order to ensure that all recycling facilities receive solid waste, guarantying this way the jobs of deprived people in the different city areas. Equity can also be addressed when defining routes that minimize the maximum route length or minimize the difference between the longest and the shortest route lengths. Pasia et al. [21,22], Jozefowiez et al. [23,24] and Reiter and Gutjahr [25] study such problem, the so-called vehicle routing problem with route balancing (VRPRB), where two conflicting objectives are addressed: minimization of the total travel cost (or total tour length) and minimization of the maximum route length.

As referred by Garetti and Taisch [26], when only two dimensions are accomplished the system is said to be viable (economic with environment), equitable (economic with social) or bearable (environment with social) (see Fig. 1).

Under this framework, the designing, planning and operation of sustainable logistics systems, i.e., systems that take a position on economic prosperity, environmental quality and social justice are almost inexistent. The present paper aims to contribute to the reduction of this existent gap and aims to support tactical and operational planning decisions in logistics systems in order to make them sustainable: building less costly, more environmental friendly and more social concerned systems.

This work was motivated by a real case study of a recyclable waste collection system, where the problem faced was on the definition of the system service areas and associated collection

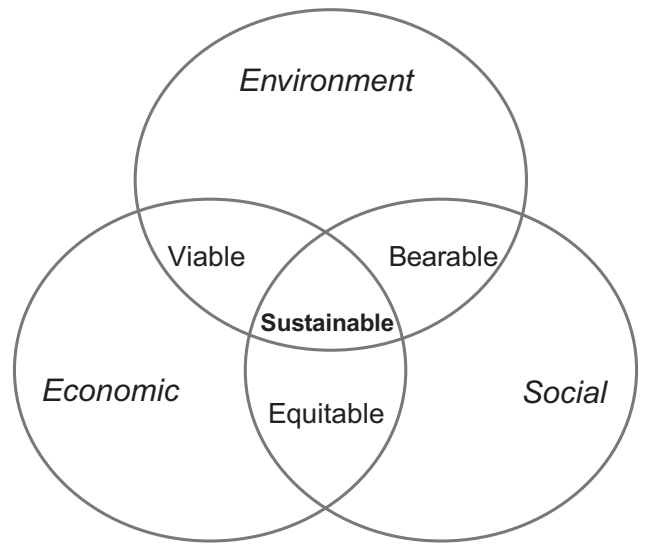

Fig. 1. The three pillars of sustainability [26]. routes that would support a sustainable solution, where not only economic objectives would be considered, but also environmental and social aspects would be accounted for. A multi-objective solution approach based on mixed-integer linear programming models is developed and applied to the case study. The economic dimension is modeled through the traveling distance that directly influences the variable cost. The environmental dimension is modeled throughout the calculations of the $\mathrm{CO}_{2}$ emissions. Finally, the social aspect is considered by aiming to define a balanced solution in terms of working hours among drivers.

The paper is organized as follows. In Section 2 we present the case study that motivated the present work. In Section 3 we review the related work. The formulation of the multi-objective problem is presented in Section 4 and the solution approach described in Section 5. Then, the results obtained for the case study are presented in Section 6. Finally, some conclusions are drawn in Section 7.

\section{Case study}

The case-study that motivated the present work is based on a recyclable packaging waste collection system that can be generally defined as a system that, within a certain geographic area and on a regular basis, collects three types of recyclable materials (glass, paper and plastic/metal) dropped by the final consumer into special containers. The involved materials are then sorted, at sorting stations, and delivered to recyclers.

In Portugal there are several collection systems in operation, each one responsible for a certain number of municipalities. Our case study focuses on the company responsible for the recyclable collection system covering 19 rural municipalities with a total area of $7000 \mathrm{~km}^{2}$. This company operates four depots and a vehicle fleet of eight vehicles. One of the depots operates also as a sorting station (depot 208). The remaining three depots are only transfer stations, where the recyclable waste is consolidated and afterwards transferred to the sorting station. The system involves 1522 glass bins, 1238 paper bins and 1205 plastic/metal bins spread over 207 localities (see Fig. 2). It is assumed that a collection site corresponds to a locality instead of an individual container in order to reduce the problem size. Due to the proximity of the containers within a locality (an average distance of $500 \mathrm{~m}$ ) it is practicable to treat the containers to collect within a locality as a single node. The number of containers at each locality/collection site is a given parameter provided by the company. Regarding the quantities to collect in each collection site, they were obtained through the analysis of the historical database of the routes

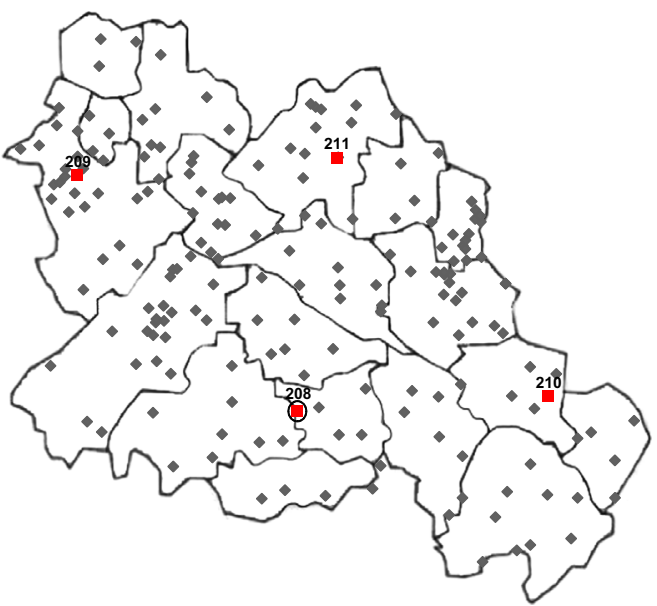

Collection site
Depot Sorting station

Fig. 2. Collection sites and depot locations. 


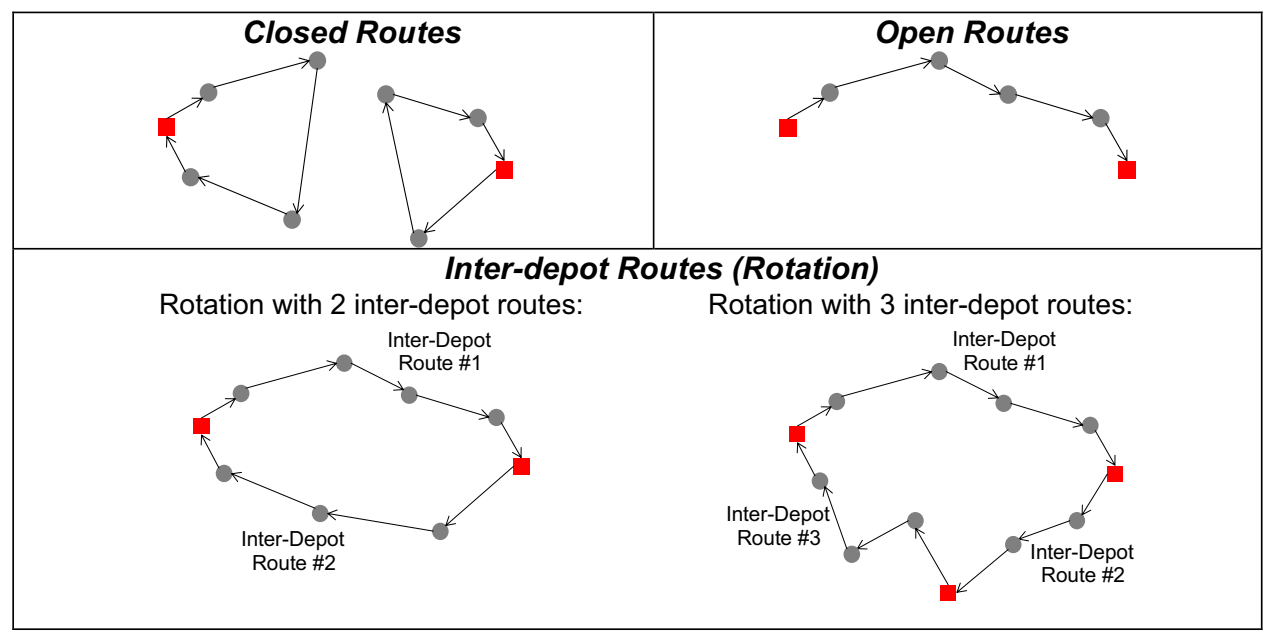

Fig. 3. Illustration of closed, open and inter-depot routes.

performed over a year. For each performed route, this database has information concerning the day, the type of recyclable material collected, the number of containers collected, the amount of kilometers traveled, the route duration and the collected weight. To estimate the amount to be collected in each collection site and the collection frequency, the daily average amount disposed by a container was determined. This estimation was based on the time interval between two consecutive collections and on the average amount collected by a container in each route.

The three recyclable materials present different collection frequencies. Glass has to be collected every 6 weeks, plastic/metal every 3 weeks and paper every 2 weeks. Therefore, a 6-week planning horizon is assumed. The materials have to be collected in separated routes since the vehicle fleet has no compartments. Due to vehicle volume capacity constraints and taking each material density into account, vehicles can load a maximum of $8500 \mathrm{~kg}$ of glass, $3000 \mathrm{~kg}$ of paper and $1000 \mathrm{~kg}$ of plastic/metal. For the outbound transportation, i.e., from the depots to the sorting station, larger vehicles are used. Weight capacities are increased to $12,000 \mathrm{~kg}$ for glass, $5000 \mathrm{~kg}$ for paper and 3000 for plastic/metal. All collection routes start at a depot, visit several localities collecting a single type of material, and return to a depot to unload. Multiple trips per day, as well as interdepot routes (routes that start and end at different depots) are allowed. However, by the end of a working day, all vehicles have to return to their origin depot. Collection is performed 5-days a week, $8 \mathrm{~h} /$ day. Till recently, all operations have been managed under a municipality-perspective, i.e., the service areas of each depot and the collection routes were defined taking into account the municipalities boundaries. This approach has proved to be very costly and motivated the restructure of the company's tactical and operational planning decisions. Moreover, the company aims to foster the system's sustainability by integrating economic, social and environmental objectives in the new plan.

The new plan should consider a vehicle route planning for a 6 week period that is to be repeated every 6 weeks. To avoid containers' overflow, route scheduling should take into account, for each material, a minimal and a maximum interval between two consecutive collections.

Regarding the economic objective, only the variable costs of the system are considered, since the fixed costs are associated with strategic decisions that had been taken beforehand (e.g. number of depots, vehicles and drivers). In this context, the variable costs are mainly associated with the distance traveled by vehicles when collecting containers and transporting the recyclable waste to the sorting station. Currently, the total distance traveled is about $270,000 \mathrm{~km} /$ year. On the environmental objective, and since transportation is this system's main activity, a concern towards Greenhouse Gas emissions in the collection routes should be taken into consideration. It was estimated that $340,000 \mathrm{~kg}$ of $\mathrm{CO}_{2}$ are emitted per year. Finally, the social objective is linked to the promotion of equity among human resources, in this case, the drivers. In the current plan, the driver's schedules are imbalanced meaning that some drivers operate excessive collection routes, while others may be idle. A maximum of 220 and a minimum of 100 driving hours are observed in a 6-week horizon. The company now wants to put into practice a solution which will account this organizational issue.

\section{Problem modeling framework and related literature}

As mentioned, the case study involves the definition and scheduling of vehicle routes in a multiple depot system, where inter-depot routes and multiple trips per vehicle are allowed. This problem is then modeled as a multi-depot periodic vehicle routing problem with interdepot routes (MDPVRPI) that consists of simultaneously selecting a set of visit days for each customer, defining the service areas of each depot and establishing multiple routes for each day of the planning horizon. The MDPVRP combines three problems: a multi-depot vehicle routing problem (MDVRP), a periodic vehicle routing problem (PVRP) and a vehicle routing problem with multiple use of vehicles (VRPMU). While the MDVRP considers a planning horizon of just one time unit, the PVRP considers a planning horizon of several time units, as it assumes that customers have different delivery (or collection) patterns. A customer specifies a service frequency and a set of allowable delivery patterns and the company has to decide on which day the delivery will occur. In the VRPMU, a vehicle can perform several routes during a working day and/or during the planning horizon. The multiple use of vehicles appears when the vehicle fleet is small or when the length of the working day is larger than the route average duration (see the works in Refs. [27-30]).

The MDVRP and the PVRP have received a great deal of attention in the literature (see, for example, the works in Refs. [31-35]), but the combination of them has been seldom studied. Consequently, only few models have been developed. Hadjiconstantinou and Baldacci [36] present a heuristic approach based on tabu search for the MDPVRP, where the boundaries of the geographic areas served by each depot are firstly determined. Parthanadee and Logendran [37] provide a model formulation, develop three tabu-search-based algorithms and propose a fast technique to find a lower bound (the selective LP relaxation) to the multi-product, MDPVRP. Vidal et al. [38] propose a hybrid genetic algorithm with adaptive diversity control for the MDPVRP. 
While in the classical MDPVRP all routes have to start and end in the same depot (closed routes), in the MDPVRP with inter-depot routes (MDPVRPI), vehicles can renew their capacity in any depot in order to continue delivering or collecting materials without being forced to return to their base depot before the end of the working day. Hence, routes can start and finish at different depots enabling a vehicle rotation composed by inter-depot routes. The concepts of closed, open and inter-depot routes are illustrated in Fig. 3. While closed routes have to start and end at the same depot, an open route ends at a different depot. The difference between an open and an inter-depot route is that in the latter a rotation has to be defined in order to get the vehicle back to its home depot. A rotation is a set of inter-depot routes that can be performed consecutively until the home depot is reached.

To the authors' best knowledge, the problem combining multiple depots, multiple periods and inter-depot routes has not yet been addressed in the literature. Nonetheless, the MDVRP with only open routes appears with a different name (non-fixed destination MDVRP) in the work of Filipec et al. [39], where it is studied and solved through a genetic algorithm. Also Ramos et al. [40] study a similar problem, the MDVRP with mixed open and closed routes (MDVRPMCO) through a MILP model approach. The MDVRP with inter-depot routes (MDVRPI) was introduced by Crevier et al. [41] and solved with a tabu search and a set partitioning algorithm. However, when solving the problem, the authors assumed that all vehicles are based at a single central depot rendering the problem as a VRP with intermediate facilities [42]. Later on, the MDVRPI with the vehicle fleet based at multiple depots is solved by Ramos [43] through a hybrid method combining heuristics with MILP formulations.

In all the above works, the objective function is defined as the minimization of either the total distance traveled or the total routing cost. Only some recent works have explored environmental issues in vehicle routing problems (Bektas and Laporte [7], Erdogan and Miller-Hooks [9]). To the best of the authors' knowledge, the integration of the three dimensions of sustainability has however never been addressed in vehicle routing problems with multiple depots. Therefore, the main contribution of this work is to propose a new solution approach to support tactical and operational decisions when planning sustainable logistics systems.

\section{Multi-objective formulation for the MDPVRPI}

The multi-objective MDPVRPI is formulated as a set partitioning problem, where $K$ represents the set of all feasible routes (closed and inter-depot routes) and $\tau_{k}$ is a binary variable that takes the value of 1 if route $k$ is part of the optimal solution. In our case, besides selecting the routes that take part of the solution, the selected routes have also to be assigned to a day $t$ of the planning horizon and to a vehicle $g$. Therefore, we redefine the assignment variable to $\tau_{k t g}$ which equals 1 if route $k$ is performed on day $t$ by vehicle $g$ and 0 otherwise.

The mathematical formulation considers the following indices and sets.

Indices

$k \quad$ Route indices

$t \quad$ Time period (days) indices

$g \quad$ Vehicle indices

i,j $\quad$ Node indices

$m \quad$ Recyclable material indices

Sets

$K \quad$ Route set $K=\underset{m \in M}{\cup} K_{m}$ and $K=K_{i n} \cup K_{c l}$

$K_{m} \quad$ Route subset to collect material $m$
$K_{\text {in }} \quad$ Inter-depot route subset

$K_{c l} \quad$ Closed route subset

$T \quad$ Time period set

$G \quad$ Vehicle set

$V \quad$ Node set $V=V_{c} \cup V_{d} \cup V_{s}$

$V_{c} \quad$ Collection site subset

$V_{d} \quad$ Depot subset

$V_{s} \quad$ Sorting station subset

M Recyclable material set

Each route $k \in K$ is characterized by (1) distance $\operatorname{dis}_{k}$; (2) duration $d u r_{k}$, which includes travel, service and unloading times; (3) load $L o_{k}$; and (4) $\mathrm{CO}_{2}$ emissions $\mathrm{Co}_{k}$. The collection sites belonging to route $k$ are given by a binary parameter $\mu_{i k}$ that equals to 1 if collection site $i$ belongs to route $k$ and 0 otherwise. The starting and ending depots for route $k$ are also given by binary parameters $S t_{k i}$ and $E n_{k i}$, respectively: $S t_{k i}$ equals to 1 if route $k$ starts at depot $i$ and $E n_{k i}$ equals to 1 if route $k$ ends at depot $i$.

The vehicles are fixed at the depots. If vehicle $g$ belongs to depot $i$, the binary parameter $\alpha_{g i}$ equals to 1 and 0 otherwise.

The collection frequency of each collection site $i$ with recyclable material $m$ is given by $f r_{i m}$ representing the number of times that a collection site has to be visited within the planning horizon. The minimum and maximum interval between two consecutive collections for recyclable material $m$ are given by $\operatorname{Imin}_{m}$ and $\operatorname{Imax}_{m}$, respectively.

Three objective functions are addressed in this work to tackle all three sustainability dimensions: economic objective $\left(z^{1}(S)\right.$ ), environmental objective $\left(z^{2}(S)\right)$ and the social objective $\left(z^{3}(S)\right)$. Let $S$ be the vector of decision variables, $z^{1}(S), z^{2}(S)$, and $z^{3}(S)$ the three objective functions and $\Omega$ the feasible region, the multiobjective problem can be written in the following form:

$$
\begin{aligned}
\min & \left\{z^{1}(S), z^{2}(S), z^{3}(S)\right\} \\
\text { st } & S \in \Omega
\end{aligned}
$$

The economic objective is measured by the collection variable costs. The fixed costs are not modeled since they correspond to decisions that were taken beforehand, such as, number of depots, number of vehicles, number of drivers, and cannot be changed. Therefore, only variable costs are considered and a common approach in routing and distribution/collection problems is used to estimate those as a function of the distance traveled. Thus, the economic objective function is here assessed by the total distance traveled. This includes the inbound distance, from the collection sites to the depots, and the outbound distance, from the depots to the sorting stations. The total distance traveled $\left(z^{1}(S)\right)$ is given by equation (2).

$$
\begin{aligned}
z^{1}(S)= & \sum_{k \in K} \sum_{t \in T g \in G} \sum_{g} d i s_{k} \tau_{k t g}+ \\
& \sum_{j \in V_{s} i \in V_{d}} \sum_{m \in M} \sum_{k \in K_{m}} \sum_{t \in T g \in G} \sum_{k} E n_{k i} \tau_{k t g} L o_{k} / Q T_{m} 2 d_{i j}- \\
& \sum_{j \in V_{s} i \in V_{d}} \sum_{m \in M} \sum_{k \in K_{m}} \sum_{t \in T} \sum_{g \in G} S t_{k i} E n_{k i} \tau_{k t g} L o_{k} / Q T_{m} 2 d_{i j}+ \\
& \sum_{\substack{g \in G \\
\alpha_{g i}=1}} \sum_{\substack{k \in K \\
E n_{k i}=0 \\
S t_{k j}=1}} \sum_{t \in T i, j \in V_{d}} 2 \tau_{k t g} d_{i j}
\end{aligned}
$$

The total distance traveled involves, as mentioned, the inbound distance (2a), the outbound distance (2b) and (2c) and also a possible extra distance as it is allowed to have vehicles based at depot $i$ to 
Total of Working Hours

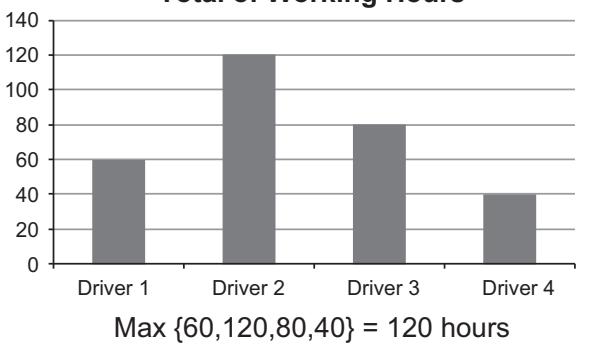

Total of Working Hours

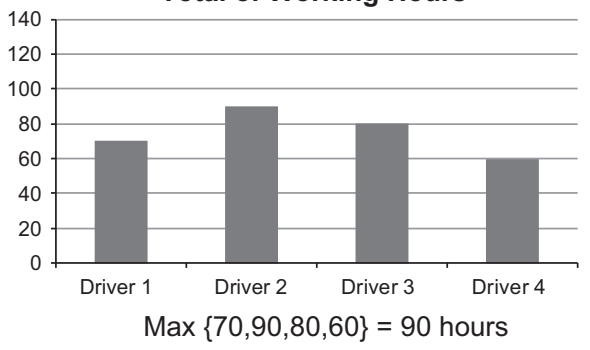

Fig. 4. Example of the effect of minimizing the maximum working hours.

perform closed routes from and to depot $j(2 \mathrm{~d})$. The distance $\left(d_{i j}\right)$ of moving a vehicle between depots is then penalized. The outbound distance considers the ending depot of each route and the load collected, in order to compute the number of needed round-trips to the sorting station. Note that the number of round-trips is not round upward as we are accounting for the number of round-trips that occur within a finite time period, but these are repeated in the next periods. When, for instance, 10.4 round-trips are considered within the time period, it means that 10 round-trips occur within the time period, and the 11th occurs in the next period, but some of the load is related to the previous period. It is also considered that if a vehicle belonging to the sorting station performs closed routes from depot $i$, the load collected will be unloaded at the sorting station and not at depot $i$. Therefore, no outbound distance will be accounted for. Term (2c) decreases the objective function of such a value.

The environmental objective is related to the $\mathrm{CO}_{2}$ emissions associated to the collection routes and to the outbound transportation between depots and the sorting station. Being a recyclable waste collection transportation activity, Greenhouse Gas emissions (GHG, like $\mathrm{CO}_{2}, \mathrm{CH}_{4} \mathrm{HFCs}, \mathrm{NO}_{x}$ ) are generated, particularly $\mathrm{CO}_{2}$ emissions, which negatively impact the environment. In this context, we measure the environmental objective through the $\mathrm{CO}_{2}$ emissions, where the total value $\left(z^{2}(S)\right)$ is given by Eq. (3).

$$
\begin{aligned}
& z^{2}(S)=\sum_{k \in K} \sum_{t \in T g \in G} \sum_{G} C o_{k} \tau_{k t g}+ \\
& \sum_{j \in V_{s} i \in V_{d}} \sum_{m \in M k} \sum_{k} \sum_{m} \sum_{t \in T} E n_{k i} \tau_{k t g} L o_{k} / Q T_{m}\left(C o F_{i j m}+C o E_{j i}\right)- \\
& \sum_{j \in V_{s} i \in V_{d}} \sum_{m \in M} \sum_{k \in K_{m}} \sum_{t \in T} \sum_{\substack{g \in G \\
\alpha_{g j}=1}} S t_{k i} E n_{k i} \tau_{k t g} L o_{k} / Q T_{m}\left(C o F_{i j m}+C o E_{j i}\right)+ \\
& \sum_{g \in G} \sum_{k \in K} \sum_{t \in T i, j \in V_{d}} 2 \tau_{k t g} \operatorname{CoE}_{i j} \\
& \alpha_{g i}=1 E n_{k i}=0 \\
& S t_{k j}=1
\end{aligned}
$$

The $\mathrm{CO}_{2}$ emissions for the inbound transportation are given by the first term (3a), where the emission value of each route $k$ is given by parameter $\mathrm{Co}_{k}$. The $\mathrm{CO}_{2}$ emissions from the outbound transportation are also considered (terms (3b) and (3c)), where larger vehicles are used. Notice that round-trips between the sorting station and the depots are performed, with vehicles traveling empty from the sorting station to the depot and in full-truck-load (FTL) back to the sorting station. The amount of $\mathrm{CO}_{2}$ emissions for outbound transportation is given by parameter $\mathrm{CoF}_{i j m}$ when the vehicle travels in FTL from depot $i$ to sorting station $j$ with material $m$ and $C_{0} E_{i j}$ when the vehicle travels empty in the opposite direction. The last term (3d) accounts for the $\mathrm{CO}_{2}$ emissions of a vehicle based at depot $i$ traveling empty to depot $j$ to perform closed routes from and to depot $j$.
Finally, the social objective is modeled through the minimization of the maximum working hours among all drivers in the planning horizon. This metric has a twofold contribution towards social sustainability. On one hand, it promotes equity amongst the drivers, enabling a balanced workload. When minimizing the maximum working hours among drivers, the under loaded drivers will be assigned to more collection routes, while the over loaded drivers will have allocated less collection routes. In this way, a more balanced workload between drivers is achieved (see Fig. 4 for an illustrative example). On the other hand, minimizing the maximum working hours releases drivers to activities other than just collection such as sorting activities, participation in recycling awareness campaigns or training. This latter activity helps to improve the career development and promotes versatility among human resources.

Assuming a fixed driver-vehicle combination, the maximum value among vehicle's total working hours in the planning horizon is given by a positive decision variable DMax (constraint (4)).

$$
\text { DMax } \geq \sum_{k \in K} \sum_{t \in T} \tau_{k t g} d u r_{k}+\sum_{\substack{k \in K \\ S t_{k j}=1 \\ E n_{k i}=0}} \sum_{\substack{i, j \in V_{d} \\ i \neq j}} \tau_{k t g} 2 b_{i j}, \quad \forall g
$$

Then, the function for the social objective is given by Eq. (5).

$z^{3}(S)=D M a x$

Having defined the objective functions, the problem constraints of the multi-objective model for the MDPVRPI are expressed in constraints (6)-(13).

$$
\begin{aligned}
& \sum_{k \in K_{m}} \sum_{t \in T} \sum_{g \in G} \tau_{k t g} \mu_{i k}=f r_{i m} \quad \forall i \in V_{c}, \forall m \\
& \sum_{k \in K} \tau_{k t g} d u r_{k}+\sum_{\substack{k \in K \\
S t_{k j}=1 \\
E n_{k i}=0}} \sum_{\substack{j \in V_{d} \\
j \neq i}} \tau_{k t g} 2 b_{i j} \leq H \quad \forall t, \forall g, \forall i \in V_{d}: \alpha_{g i}=1 \\
& \sum_{k \in K_{i n}} \tau_{k t g}=\sum_{\substack{k^{\prime} \in K_{i n} \\
E n_{k^{\prime} i}=1}} \tau_{k^{\prime} t g} \quad \forall g, \forall t, \forall i \in V_{d} \\
& S t_{k i}=1 \\
& \sum_{g \in G} \tau_{k t g} \mu_{i k}+\sum_{g \in G} \tau_{k t^{\prime} g} \mu_{i k} \leq 1 \quad \forall i \in V_{c}, \\
& \forall k \in K_{m}, \forall m, \forall t, t^{\prime} \in T, t>t^{\prime},\left(t-t^{\prime}\right) \leq \operatorname{Imin} m \\
& \sum_{g \in G} \tau_{k t g} \mu_{i k}+\sum_{g \in G} \tau_{k^{\prime} t^{\prime} g} \mu_{i k^{\prime}} \leq 1 \quad \forall i \in V_{c}, \forall k, k^{\prime} \in K_{m}, \forall m, \forall t, t^{\prime} \in T \\
& t>t^{\prime},\left(t-t^{\prime}\right) \leq \operatorname{Imin}_{m} \\
& \sum_{g \in G} \tau_{k t g} \mu_{i k}+\sum_{g \in G} \tau_{k t^{\prime} g} \mu_{i k} \leq 1 \quad \forall i \in V_{c}, \forall k \in K_{m}, \forall m, \forall t, t^{\prime} \in T \\
& t>t^{\prime},\left(t-t^{\prime}\right)>\operatorname{Imax}_{m},\left(t-t^{\prime}\right) \leq \operatorname{Imax} m+\operatorname{Imin}_{m}
\end{aligned}
$$




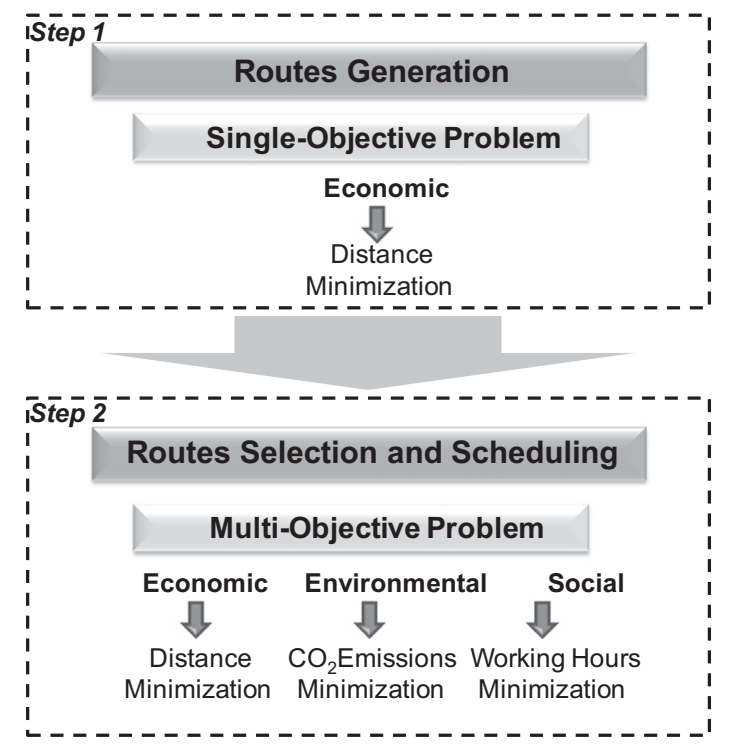

Fig. 5. Solution approach overview.

$\sum_{g \in G} \tau_{k t g} \mu_{i k}+\sum_{g \in G} \tau_{k^{\prime} t^{\prime} g} \mu_{i k^{\prime}} \leq 1 \quad \forall i \in V_{c}, \forall k, k^{\prime} \in K_{m}, \forall m, \forall t, t^{\prime} \in T$,

$t>t^{\prime},\left(t-t^{\prime}\right)>\operatorname{Imax}_{m},\left(t-t^{\prime}\right) \leq \operatorname{Imax}_{m}+\operatorname{Imin}_{m}$

$\tau_{k t g} \in\{0,1\} \quad \forall k \in K, \forall t \in T, \forall g \in G$

Constraint (6) ensures that a collection site $i$ with material $m$ has to be collected $f r_{i m}$ times over the time horizon. Constraint (7) states that the total route duration performed by vehicle $g$ on day $t$ will not exceed the maximum time allowed for a working day $(H)$. If a vehicle $g$, belonging to depot $i$, performs a route starting at depot $j$, the travel time between $i$ and $j\left(b_{i j}\right)$ is considered.

Considering that all vehicles have to return to their origin depot, constraint (8) guarantees that an inter-depot route $k$ starting at depot $i$ is part of the solution only if another interdepot route $k^{\prime}$ ends at depot $i$. Considering all depots $i \in V_{d}$, constraint (8) ensures continuity among inter-depot routes enabling vehicle rotation.

Constraints (9)-(12) model the minimum and maximum intervals between consecutive collections which can be performed by the same route or by two different routes. Therefore, constraint (9) states that the same route for material $m$ has to be performed with a minimum time interval of $\operatorname{Imin}_{m}$ while constraint (10) considers the case of two different routes collecting the same site $i$, at consecutive collections. Analogously, constraints (11) and (12) ensure the maximum interval $\operatorname{Imax}_{m}$ between consecutive collections. Variable's domain is given in constraint (13).

\section{Solution approach}

To solve the multi-objective MDPVRPI formulated in the previous section a solution approach is developed. Since the problem is modeled with the set partitioning formulation, a procedure to generate the set $K$ of feasible routes is needed beforehand. Therefore, our solution approach involves a first step to generate the routes and a second step where the multi-objective problem is solved (see Fig. 5). As the goal is to obtain a solution where the costs are balanced with environmental and social concerns, the set of routes $K$ is defined considering only the economic objective. When selecting and scheduling the routes, at step 2 , the three objectives are taken into account by solving the multi-objective MDPVRPI through the augmented $\varepsilon$-constraint method. With such

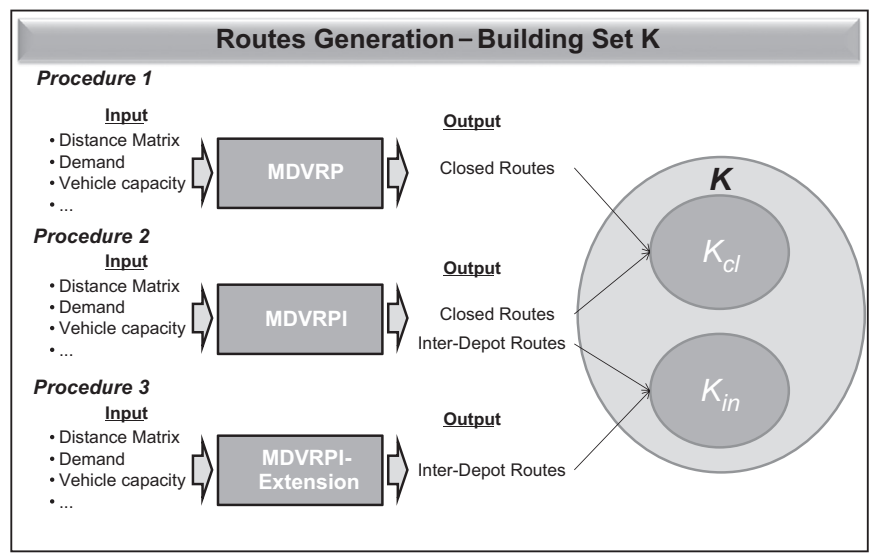

Fig. 6. Routes generation procedures.

method, an approximation to the Pareto front is obtained, which can be used by the decision-maker to evaluate trade-offs and to select the solution to be adopted.

Each step of the solution approach is detailed in the next sections.

\subsection{Step 1 - routes generation}

As mentioned, the goal of step 1 is to build the set of feasible routes $K\left(K=K_{i n} \cup K_{c l}\right)$ required by the multi-objective MDPVRPI formulation. Generating all the feasible routes is however intractable (Laporte [44]), so only a subset of routes will be defined. Regarding the characteristics of the addressed problem, a diverse set of closed and inter-depot routes are generated representing alternative solutions to collect all sites. To build only closed routes $\left(K_{c l}\right)$, a MDVRP is solved. To build closed $\left(K_{c l}\right)$ and inter-depot $\left(K_{i n}\right)$ routes, a MDVRPI is solved. To build only interdepot routes $\left(K_{i n}\right)$, a MDVRPI-Extension is solved. Therefore, set $K$ is fed by three independent procedures modeling the three alternative solutions to collect waste from all collection sites (see Fig. 6).

We recall that as we are dealing with a multi-product problem, a set of recyclable materials $M$ is involved, and given that each material has to be collected in separated routes, each procedure of step 1 is run independently for each material.

The problems involved in each procedure are formulated through MILP formulations based on the two-commodity flow formulation [45]. In such formulations, the network is defined by a direct graph $G R=(V, E)$ with $V=V_{c} \cup V_{d} \cup V_{f} \cup V_{s}$, being $V_{c}=\{1, \ldots, n\}$ a set of $n$ customers, $V_{d}=\{n+1, \ldots, n+w\}$ a set of $w$ depots, $V_{f}=\{n+w+1, \ldots, n+2 w\}$ a replica of the depots set, $V_{s}=$ $\{n+2 w+1, \ldots, n+2 w+s\}$ a set of $s$ sorting stations and $E=\{(i, j): i$, $\left.j \in V_{c} \cup V_{d} \cup V_{f} \cup V_{s}, i \neq j\right\}$ the edge set.

Each site $i \in V_{c}$ is characterized by a demand $p_{i}$ and service duration $t_{i}$. The service duration depends on the average time to collect a container $(U)$, on the average distance between containers within a locality $(B)$, on the average speed within localities $(\nu w)$ and on the number of containers at each locality $\left(c_{i}\right)$, where $t_{i}=c_{i}(U+(B / v w))$. The inbound vehicles have a weight capacity of $Q$ and the outbound vehicles $Q T$. The maximum duration for a working day is given by $H$. Every edge $(i, j)$ has an associated distance $d_{i j}$ and a travel time $b_{i j}$, where $b_{i j}=\left(d_{i j} / v b\right)$ and $v b$ is the average speed between localities. We assumed the same speed in every edge as the type of roads and traffic in the studied rural network are very similar. Moreover, only ordinary roads between localities are traveled by these vehicles, so it is a reasonable assumption to consider same speed in every edge. An unloading 
time $L$ is also considered to account for the time to unload a vehicle at the end of each route.

The depot replica set $\left(V_{f}\right)$ is needed because in the twocommodity flow formulation routes are defined by paths starting at the real depots and ending at the replica ones. To establish the routes, this formulation requires two flow variables defining two flow paths for any route. One path from the real depot to the replica one modeled by the flow variable representing the vehicle load (variable $y_{i j}$ ). In a collection problem, this load increases along the route. The other path, from the replica depot to the real one, is given by the second flow variable $\left(y_{j i}\right)$ that models the vehicle empty space which decreases along the route.

These sets, parameters and variables are the baseline to all routes generation procedures which are briefly described in the next sections.

\subsubsection{Procedure 1 - MDVRP}

In the MDVRP only closed routes are defined. A set of routes $K$ is considered and partitioned by depot: $K=K_{1} \cup \ldots \cup K_{i}$, where $K_{i}$ is the subset of routes belonging to depot $i$. As decision variables we have the binary variable $x_{i j k}$ that equals to 1 if site $j$ is visited immediately after site $i$ on route $k\left(x_{i j k}=0\right.$, otherwise) and the corresponding reverse variable $x_{j i k}$ when the reverse path is being defined; the flow variables $y_{i j k}$ and $y_{j i k}$; and a binary variable $\delta_{i k}$ is defined to assign site $i$ to route $k$. The objective function also considers the distance to be traveled within each collection site (second term of Eq. (14)) and the outbound distance (third term of Eq. (14)).

$\operatorname{Min} \frac{1}{2} \sum_{i \in V} \sum_{j \in V} \sum_{k \in K} x_{i j k} d_{i j}+\sum_{i \in V_{c}} c_{i} B+2 \sum_{i \in V_{c}} \sum_{j \in V_{f}} \sum_{h \in V_{s}} \sum_{k \in K} \frac{y_{i j k}}{Q T} d_{h j}$

subject to

$\sum_{j \in V}\left(y_{i j k}-y_{j i k}\right)=2 p_{i} \delta_{i k}, \quad \forall i \in V_{c}, \forall k$

$j \neq i$

$\sum_{i \in V_{c}} \sum_{j \in V_{f}} \sum_{k \in K} y_{i j k}=\sum_{i \in V_{c}} p_{1}$

$\sum_{i \in V_{c}} \sum_{j \in V_{f}} \sum_{k \in K} y_{j i k} \leq|K| Q-\sum_{i \in V_{c}} p_{i}$

$\sum_{i \in V_{c}} y_{i j k} \leq Q \quad \forall_{j} \in V_{f}, \forall k \in K_{j}$

$\sum_{i \in V} x_{i j k}=2 \delta_{j k}, \quad \forall j \in V_{c}, \forall k$

$i \neq j$

$y_{i j k}+y_{j i k}=Q x_{i j k} \quad \forall i, j \in V, i \neq j, \forall k$

$\sum_{k \in K} \delta_{i k}=1 \quad \forall i \in V_{c}: p_{i}>0$

$\delta_{i k}=\delta_{(i+w) k} \quad \forall i \in V_{d}, \forall k \in K_{i}$

$\sum_{i \in V_{c}} \sum_{j \in V} t_{i} x_{i j k}+\sum_{i \in V} \sum_{j \in V} b_{i j} x_{i j k} \leq 2(H-L) \quad \forall k \in K$

$\sum_{j \in V_{c}} x_{i j k} \leq 1 \quad \forall i \in V_{d}, \forall k \in K_{i}$

$\sum_{i \in V_{c}} x_{i j k}=0 \quad \forall j \in V_{f}, \forall k \notin K_{j}$

$\sum_{j \in V_{c}} x_{i j k}=0 \quad \forall i \in V_{d}, \forall k \notin K_{i}$

$y_{i j k} \geq 0 \quad \forall i, j \in V, k \in K$

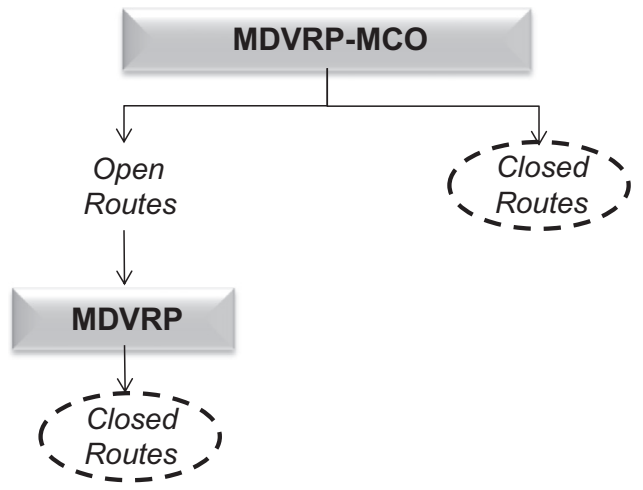

Fig. 7. Solution method for the MDVRP.

$x_{i j k} \in\{0,1\} \quad \forall i, j \in V, k \in K$

$\delta_{i k} \in\{0,1\} \quad \forall i \in V_{c}, k \in K$

The above formulation is an extension for the MDVRP of the formulation proposed by Baldacci et al. [45] for the CVRP. Constraints (15)-(20) are re-written as we now consider index $k$ and the binary variable $\delta_{i k}$. Constraints (21)-(26) are new constraints that deal with multiple depots and duration constraints. Constraint (21) guarantees that each locality with positive demand has to be visited by a single route. Constraint (22) matches the real depots with their replica, ensuring that a route will start at real depot and will end at the corresponding replica. Constraint (23) guarantees that the duration of each route does not exceed the maximum allowed routing time. Constraint (24) ensures that each route will leave its home depot at most once. Finally, constraints (25) and (26) jointly ensure that a vehicle route cannot leave and return to a depot other than its home depot (real and replica depot). The new variable definition is given in Eq. (29).

The proposed formulation when applied to large instances is difficult to solve. Therefore, a solution method is proposed to solve the MDVRP (see Fig. 7). Firstly, one solves a problem where both closed and open routes are allowed, the MDVRP with mixed closed and open routes (MDVRP-MCO). The MDVRP-MCO formulation is proposed in the work of Ramos et al. [40] and is capable of solving large instances. Moreover, a great part of the routes in the solution for the MDVRP-MCO are feasible for the MDVRP - the closed routes. For the infeasible part of the solution (the open routes), the MDVRP formulation is applied, having as input data only the sites belonging to each open route.

\subsubsection{Procedure 2 - MDVRPI}

The MDVRPI also allows inter-depot routes, where vehicles have to return to the home depot on the same working day. Therefore, vehicle rotation is limited by the maximum duration of a working day $(H)$. To solve the MDVRPI, we use the solution methodology proposed by Ramos [43], considering an unlimited vehicle fleet. A MDVRPI Relaxation is firstly solved where interdepot and closed routes are obtained (see Fig. 8). This formulation corresponds to the MDVRP-MCO formulation to which constraint (30) is added.

$\sum_{j \in V} x_{i j}+\sum_{j \in V} x_{j i}=\sum_{j \in V} x_{(i+w) j}+\sum_{j \in V} x_{j(i+w)} \quad \forall i \in V_{d}$

Constraint (30) guarantees that the number of routes departing from one depot is equal to the number of routes arriving at that depot. This ensures connectivity between the inter-depot routes and the rotation concept, i.e., a vehicle returns to its home depot. However, it is not guaranteed that the vehicle returns within a working day since no duration constraints for rotation are considered in the MDVRPI Relaxation. Notice that in the 


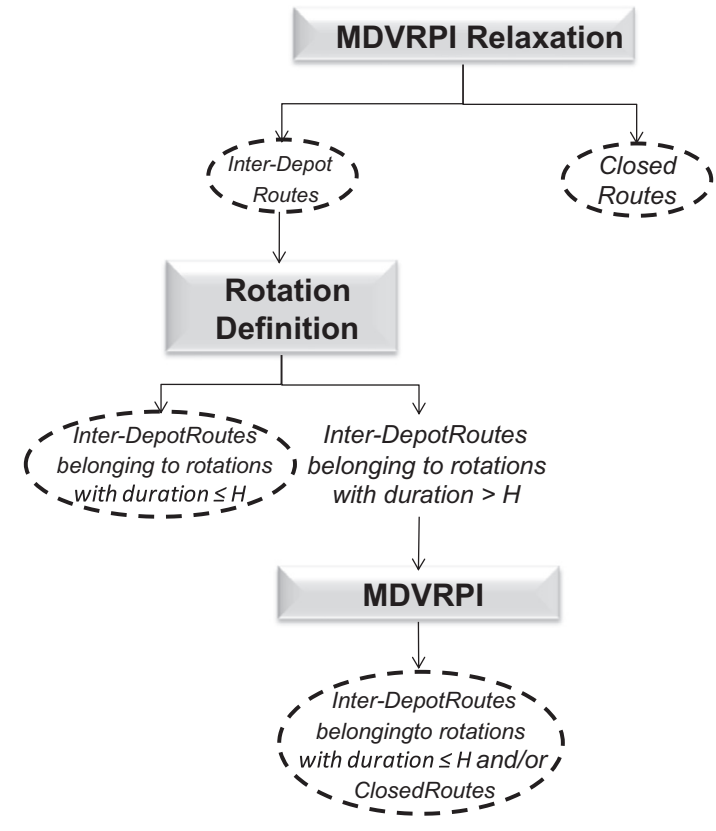

Fig. 8. Solution method for the MDVRPI.

two-commodity formulation, to any real depot $i \in V_{d}$, a corresponding replica depot is assumed $i+w \in V_{f}$ ( $w$ is the number of depots),

and $x_{i j}$ and $x_{j i}$ model the opposite paths.

For the inter-depot routes obtained from the solution of the MDVRPI Relaxation, rotations are defined by linking the interdepot routes until one reaches the starting depot. The duration of each rotation is then assessed. For rotations that do not respect the working day time limit $(H)$, the MDVRPI formulation is solved and rotations are re-defined to comply with the imposed limit. As a solution, one can have inter-depot routes belonging to rotations that satisfy the maximum duration for a working day and/or closed routes. More details can be found in Ramos [43].

\subsubsection{Procedure 3 - MDVRPI extension}

The MDVRPI Extension solves the problem by visiting all sites only by inter-depot routes. For that, the MDVRPI Relaxation is used but, instead of considering all depots and all collection sites at the same time, only two depots are considered in each run and only the closest sites to those depots are made available to be collected. Moreover, a constraint is added to enforce routes to start and end at different depots. As a result, only inter-depot routes are defined.

A pair of depots $\left[d p, d p^{\prime}\right] \in V_{d}$ is considered at a time and constraints (31) and (32) are added to the MDVRPI Relaxation formulation, imposing that all routes have to start at depot $d p$ and end at depot $d p^{\prime}$ so as to obtain a solution with only inter-depot routes between each pair of depots.

$x_{i j}=0, \quad \forall i \in V_{c}, j=d p+w$

$x_{i j}=0, \quad \forall j \in V_{c}, \quad i=d p^{\prime}$

Regarding the maximum duration for each inter-depot route in this procedure, the value $\left(H-L-b_{d p, d p^{\prime}}\right)$ is considered to guarantee that the vehicle can return to the origin depot within a working day.

After running the three procedures, set $K$ is built. Each route $k \in K$ is characterized by mileage $\left(d i s_{k}\right)$, duration $\left(d u r_{k}\right)$, load $\left(L o_{k}\right)$ and $\mathrm{CO}_{2}$ emissions $\left(\mathrm{Co}_{k}\right)$. The first three parameters are provided by the solutions of the problems solved. The last one, the $\mathrm{CO}_{2}$ emissions, has to be assessed a posteriori. For that, we use the vehicle emissions model proposed by Barth et al. [46]. This work assumes that when a vehicle travels over an arc $(i, j)$ it emits a certain amount of $\mathrm{CO}_{2}$, which depends on the fuel consumption that, in turn, is function of many aspects such as: distance traveled, vehicle load (curb weight plus load), speed, road angle, engine features, vehicle frontal surface area, coefficients of rolling resistance and drag, air density, among others (see Barth et al. [46]). To convert fuel into $\mathrm{CO}_{2}$ emissions, we assumed the conversion factor of one liter of diesel fuel containing $2.6676 \mathrm{~kg}$ of $\mathrm{CO}_{2}$ (as proposed in [47]). Note that we considered $\mathrm{CO}_{2}$ emissions on edges and nodes, since nodes represent collection sites aggregating one or more containers and a certain mileage is traveled within each node.

The computation of the $\mathrm{CO}_{2}$ emissions for all routes $k \in K$ concludes step 1.

\subsection{Step 2 - solution method for the multi-objective problem}

In step 2 the multi-objective problem defined in Section 4 is solved. In such problems is rarely the case where a single point optimizes simultaneously all the objective functions [48], therefore, trade-offs between the objectives have to be analyzed in line with the notion of Pareto optimality. A solution is Pareto-optimal if no feasible solution exists which would improve some objective without causing a simultaneous deterioration in at least one other objective. This concept generally does not provide a single solution, but rather a set of solutions called the Pareto-optimal set. The image of the Pareto-optimal set under the objective functions is called Pareto front [48].

Several methods exist to solve this kind of problems. According to Hwang and Masud [49], the multi-objective methods are classified as a priori, interactive and a posteriori, depending on when the decision-maker preferences are provided. In a posteriori methods, the decision-maker analyzes the Pareto-optimal solutions that were previously generated and takes the final decision. The most widely used a posteriori methods are (1) the weighting method, where the weighted sum of the objective functions is optimized and by varying the weights different Pareto-optimal solutions are obtained; and (2) the $\varepsilon$-constraint method, where one objective function is optimized and the other objectives are considered as constraints bounded by some allowable levels $\varepsilon$. The levels $\varepsilon$ are then altered to generate the entire Pareto-optimal set. The $\varepsilon$-constraint method has been successfully applied to routing and distribution problems (Berube et al. [50], Grandinetti et al. [51], and Liu and Papageorgiou [52]). For a detailed survey about multi-objective problems see [53,54].

An improved version of the traditional $\varepsilon$-constraint method is applied to our problem so that the Pareto front is generated. According to Mavrotas [55], the conventional $\varepsilon$-constraint method presents three points that need careful attention when implementing such a method: (1) the calculation of the range of the objective functions over the efficient set; (2) the guarantee of efficiency of the obtained solution, and (3) the increased solution time for problems with more than two objective functions. Mavrotas [55] proposes an improved version of the $\varepsilon$-constraint method to address those aspects, the so-called augmented $\varepsilon$ constraint method. To overcome the first issue, Mavrotas [55] proposes a lexicographic optimization over every objective function in order to compute the range of the objective functions (the payoff table) over the efficient set. The lexicographic approach will ensure the Pareto optimality by optimizing a first objective function and then, among the possible alternative optima, optimizing for a second objective function and so on. If the range of the objective functions is obtained only by individual optimization, it is not guaranteed that the solutions obtained are Pareto-optimal solutions since alternative optima may be presented (weak efficiency). 
To overcome the second issue, Mavrotas [55] proposes that the objective function constraints are transformed into equalities (instead of inequalities as in the conventional method) by incorporating slack or surplus non-negative variables. These new variables are then used as a second term in the objective function penalizing the objective function if their value differs from zero. This strategy forces the model to produce only efficient solutions.

When dealing with three objective functions, a total of $\left(q_{2}+1\right)$ $\left(q_{3}+1\right)$ runs are performed to obtain the Pareto front, if $q_{2}$ and $q_{3}$ are the equal intervals dividing the range of each objective function. To overcome the third issue and decrease the number of runs, the algorithm initiates with the more relaxed version of the constrained objective function and gradually restricts the bounds. When the problem becomes infeasible, it means that there is no need to further restrict the corresponding objective function as it will also result in infeasible solutions. Therefore, the algorithm proceeds to the next grid point. For further insights on the augment $\varepsilon$-constraint method please see [55].

When solving the problem under analysis in this paper, where three objectives are being tackled, an approximation to the Pareto front is designed by using the augmented $\varepsilon$-constraint method, where the economic objective is optimized and the social and environmental objectives constrained (see Table 1 ).

Finally, to propose a sustainable solution, that is, a compromise solution between the three objectives, the compromise solution method [56] is applied, where the Pareto-optimal solution closest

Table 1

Pseudo-code of the augment $\varepsilon$-constraint method.

1. Lexicographic optimization to create the payoff table

$1.1 \min z^{1}(S)$

st

Eqs. (4), (6)-(13)

Output: solution $s_{1}=\left(z^{1^{*}}, z^{2}, z^{3}\right)$

$1.2 \min z^{2}(S)$

st

Eqs. (4), (6)-(13) $+z^{1}(S)=z^{1^{*}}$

Output: solution $s_{2}=\left(z^{1^{*}}, z^{2^{*}}, z^{3}\right)$

$1.3 \min z^{3}(S)$

st

Eqs. (4), (6) $-(13)+z^{1}(S)=z^{1^{*}}+z^{2}(S)=z^{2^{*}}$

Output: solution $s_{3}=\left(z^{1^{*}}, z^{2^{*}}, z^{3^{*}}\right)$

1.4 Repeat (1.1) to (1.3) for $z^{2}(S)$ and $z^{3}(S)$

1.5 Determine the payoff table for the three objectives

2. Set $\varepsilon$ values

2.1 Set ranges of the objective functions:

$r_{2}=z_{\max }^{2}-z_{\min }^{2}$

$r_{3}=z_{\max }^{3}-z_{\min }^{3}$

2.2 Set number of grid points $q_{2}$ and $q_{3}$

2.3 Set the variation of $\varepsilon_{2}$ and $\varepsilon_{3}$ :

$\Delta \varepsilon_{2}=r_{2} / q_{2} \Delta \varepsilon_{3}=r_{3} / q_{3}$

3. Solve problem (where $v_{2}, v_{3}$ are the surplus variables and eps is a small number, usually between $10^{-3}$ and $10^{-6}$ )

$n_{2}=0, n_{3}=0$

while $n_{2} \leq q_{2}$ and $n_{3} \leq q_{3}$

do

$\min \left(z^{1}(S)-\operatorname{eps}\left(v_{2} / r_{2}+v_{3} / r_{3}\right)\right)$

st

Eq. (4), (6)-(13)

$z^{2}(S)+v_{2}=z_{\max }^{2}-n_{2} \Delta \varepsilon_{2}$

$z^{3}(S)+v_{3}=z_{\max }^{3}-n_{3} \Delta \varepsilon_{3}$

$n_{2}=n_{2}+1$

$n_{3}=n_{3}+1$

end to the ideal point is obtained. The ideal point $\left(z_{I}\right)$ is defined according to the individual minima of each objective $\left(z_{I}=\left(z_{\min }^{1}\right.\right.$, $\left.z_{\min }^{2}, z_{\min }^{3}\right)$ ), while the nadir point $\left(z_{N}\right)$ is defined according to the worst values obtained for each objective $\left(z_{N}=\left(z_{\max }^{1}, z_{\max }^{2}, z_{\max }^{3}\right)\right)$. In order to apply this method, the objective functions are normalized by the differences between the nadir and ideal points, measuring the variability of the objective function within the Pareto set. Afterwards, the compromise solution is obtained by minimizing the distance from the Pareto front to the ideal point, where the Tchebycheff norm is used as a distance measure:

$\min \left\{\max _{j=1, \ldots, \phi}\left\{\lambda_{j}\left|z^{j}(S)-z_{I}^{j}\right|\right\}: S \in \Omega\right\}$

where $\phi$ is the number of objective functions in study and $\lambda_{j}$ the normalized factor for each objective function:

$\lambda_{j}=\frac{1}{r_{j}}\left[\sum_{i=1}^{\phi} \frac{1}{r_{i}}\right]^{-1}$

$r_{j}=z_{\text {max }}^{j}-z_{\text {min }}^{j}$

\section{Results and analysis}

In this section the solution approach proposed is applied to the described case study in order to define a sustainable plan for the recyclable waste collection in 19 Portuguese municipalities. The solution approach was implemented in GAMS 23.7 and solved through the CPLEX Optimizer 12.3.0, on an Intel Xeon CPU X5680 @ $3.33 \mathrm{GHz}$.

\subsection{Results for step 1}

In step 1, a set of diverse closed and inter-depot collection routes for the three recyclable materials are generated through the run of the three described procedures. The number of routes obtained from each procedure for each material is shown in Table 2.

It can be seen that the mixture plastic/metal, which acts as a single material, requires more collection routes than the other two materials. This mixture has a lower density when compared to the other two materials, and thus the vehicle weight capacity for such material is smaller for the same vehicle volume capacity.

In order to illustrate the procedure's results, the routes obtained for the glass collection are provided in Figs. 9-11 Fig. 9(a) shows the 39 closed routes designed by procedure 1 , where a MDVRP is solved. Fig. 9(b) shows the partial results for procedure 2, where 26 closed routes and nine inter-depot routes are defined by the MDVRPI Relaxation. To complete procedure 2, the nine inter-depot routes are assigned to four rotations and the total duration of each rotation is assessed. All proposed rotations exceeded the maximum time allowed for a working day. Thus, the inter-depot routes belonging to each rotation are re-worked by the MDVRPI formulation in order to satisfy the duration constraints. Fig. 10(a) and (b) shows two of the four rotations before and after being re-worked.

Procedure 3 defines 38 inter-depot routes for glass considering two depots at each run. To select the sites for each run, the closest and the second closest depots for each site are determined. Then, for each pair of depots, one selects the sites that have these two depots as the closest and second closest depots. The result of this process is shown in Fig. 11. For 31 collection sites, marked with triangles, the closest and the second closest depots are depots 208 and 209; for pair [208-210] there are 40 sites; for depot pair [208211] there are 46 sites; for pair [209-211] there are 54 sites and for pair [210-211] there are 36 sites (see Fig. 11). 
Procedure 3 uses the MDVRPI Extension for each pair of depots and the inter-depot routes are then obtained. In Fig. 11, the eight glass inter-depot routes obtained for pair [208-211] can be seen as an illustrative example.

To sum up, a set of 97 different routes to collect glass have been defined in step 1 . The same procedure is applied to the other two materials and, as final results, 95 routes to collect paper and 145 routes to collect plastic/metal are obtained. A total of 337 routes are defined for the three types of materials. Considering that the routes obtained by procedure 3 may start at any of the two depots, these 140 routes are replicated by reverting direction and added

Table 2

Number of routes defined by procedure and by recyclable material.

\begin{tabular}{lccc}
\hline & Glass & Paper & Plastic/metal \\
\hline $\begin{array}{l}\text { Procedure 1 } \\
\text { Closed routes }\end{array}$ & 39 & 42 & 66 \\
Procedure 2 & & & \\
Closed routes & 37 & 41 & 64 \\
Inter-depot routes & 9 & 6 & 9 \\
Procedure 3 & 38 & 40 & 62 \\
Inter-depot routes & 38 & & \\
\hline
\end{tabular}

to set $K$. Moreover, direct routes between depots are also added (10 direct routes, considering both directions). In conclusion, 487 routes compose set $K$.

\subsection{Results for step 2}

Step 2 selects routes from set $K$, while considering the number of vehicles available (eight in total) and where they are based. It also accounts for the planning horizon of 6 weeks (i.e. 30 working days) and observes the interval between collections. At step 2, the multi-objective problem is solved by applying firstly the augmented $\varepsilon$-constraint method to define an approximation to the Pareto front, and then the compromise solution method to obtain a sustainable solution for the case study.

The payoff table generated by the lexicographic method is shown in Table 3. When minimizing the total distance (economic objective $z^{1}$ ), a solution with $27,261 \mathrm{~km}$ is obtained. This solution emits $34,982 \mathrm{~kg}$ of $\mathrm{CO}_{2}$ and the maximum number of hours among the eight vehicles is $200 \mathrm{~h}$. When minimizing the $\mathrm{CO}_{2}$ emissions (environmental objective $z^{2}$ ), a solution with $34,747 \mathrm{~kg}$ of $\mathrm{CO}_{2}$ is achieved. This solution implies $0.7 \%$ less of $\mathrm{CO}_{2}$ emissions, and $0.3 \%$ more kilometers when compared to the economic solution. The number of working hours remains unaltered. Lastly, when minimizing the maximum number of working hours in the planning horizon (social objective $z^{3}$ ), a solution with a maximum

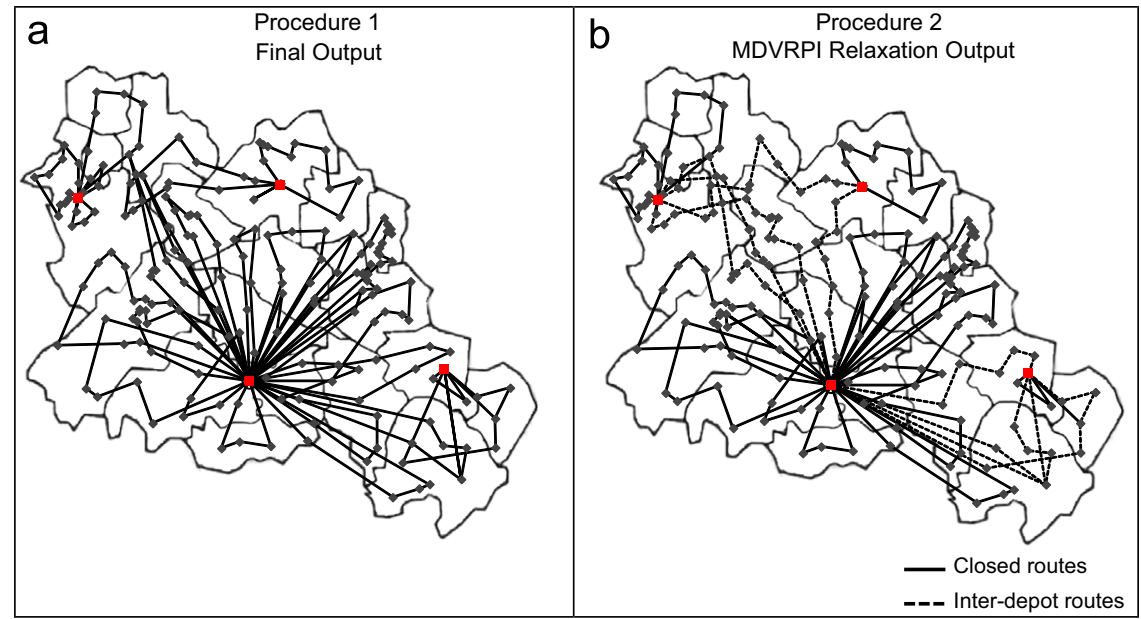

Fig. 9. Final solution for procedure 1 and partial solution for procedure 2 for material glass.

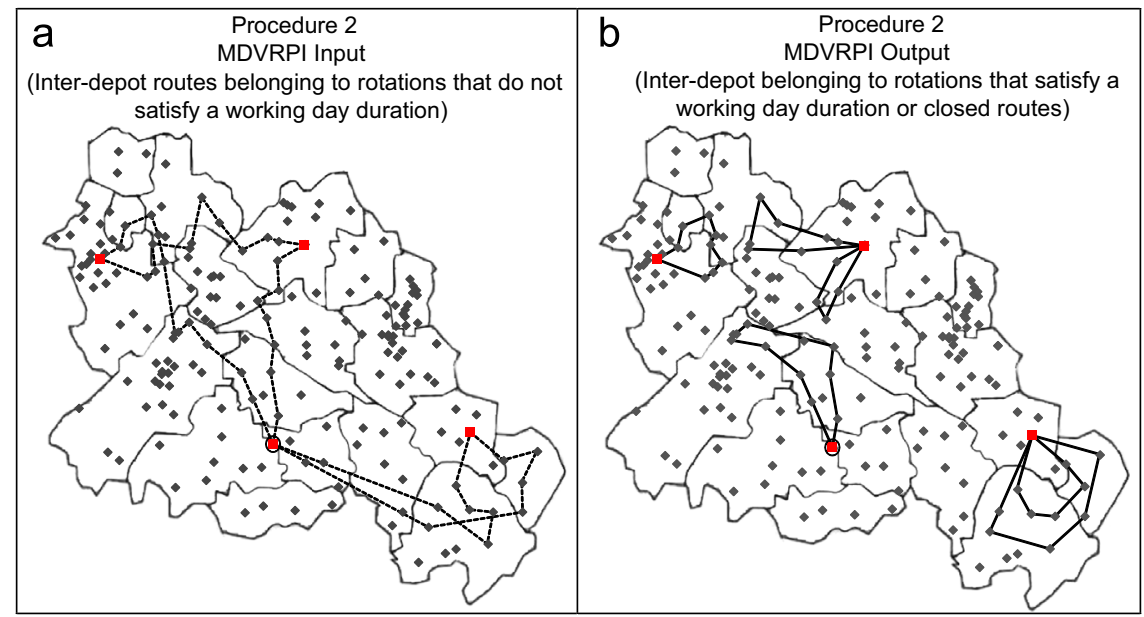

Fig. 10. Procedure 2 - MDVRPI partial solution for material glass. 


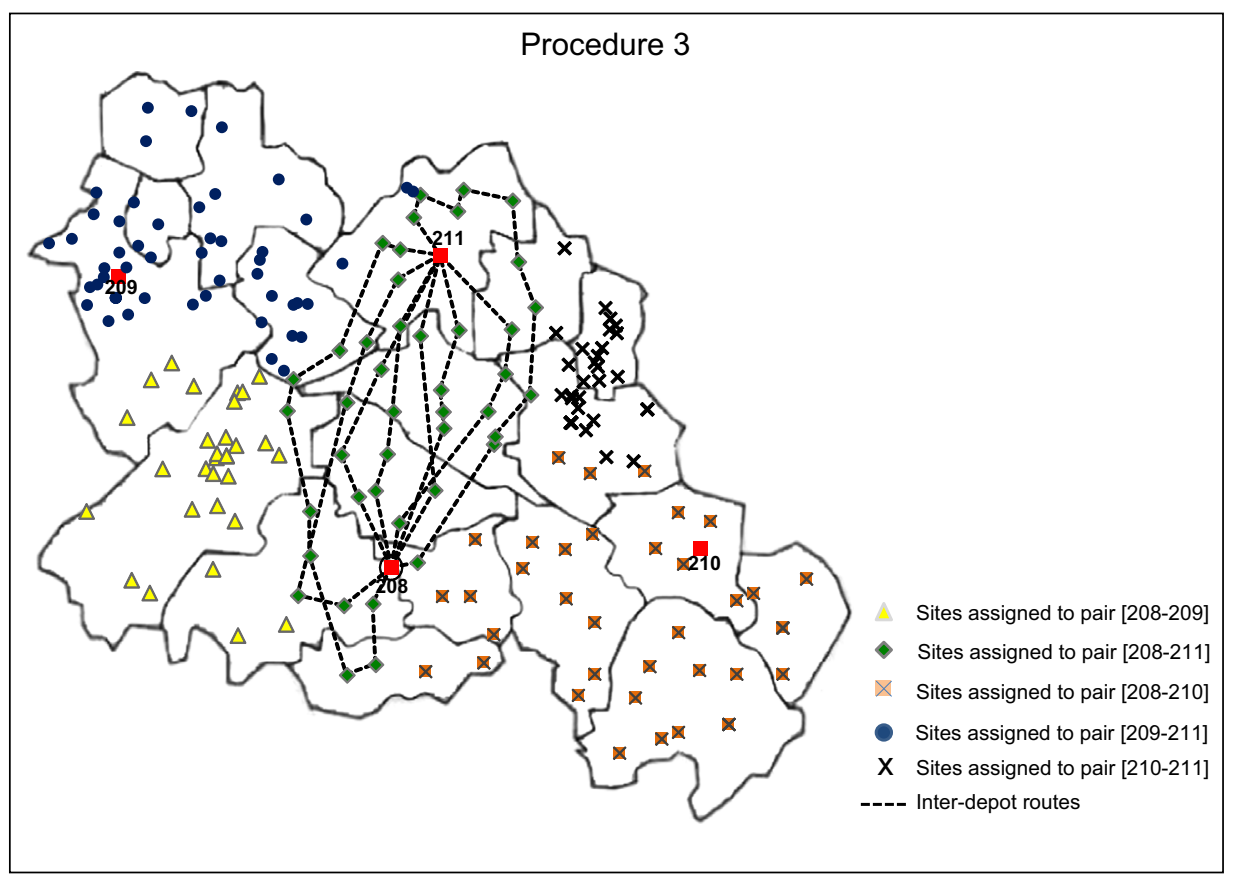

Fig. 11. Collection sites assigned to each pair of depots and the inter-depot routes for pair [208-211] provided by procedure 3.

of $165 \mathrm{~h}$ is obtained. This solution implies a total of $30,118 \mathrm{~km}$ (about 11\% more than in the economic solution) and 38,042 $\mathrm{kg}$ of $\mathrm{CO}_{2}$ (about $10 \%$ more than in the environmental solution).

When analyzing in detail the results of the payoff table, Fig. 12 shows the number of hours each driver has to work (social concern) in the collection activity considering the economic, environmental and social solutions. It can be seen that both economic and environmental solutions are quite unbalanced, with a difference between the maximum and minimum working hours of 102 and $120 \mathrm{~h}$, respectively. On the other hand, the social objective presents an equity solution, where all drivers work the same number of hours in the collection activity (165 h).

The solution obtained in step 2 is a daily schedule for each vehicle, defining all routes to be operated. Fig. 13 shows, as an example, the schedules proposed for vehicle 7 that belongs to depot 210, when considering the economic and social solutions.

Each day of the schedule shows the number and type of routes to be performed ( $\mathrm{Pl}$ stands for plastic/metal, Gl for glass and $\mathrm{Pa}$ for paper) and the total duration (in minutes). For example, on day 1 of the economic solution, vehicle 7 has to perform route \#56 to collect plastic/metal and afterwards route \#250 to collect paper. The total duration (including unloading activities) is $461 \mathrm{~min}$. Route \#250 is repeated three times over the planning horizon given the collection frequency for material paper (days 1, 12, and 22). The interval between consecutives visits respects the minimum and maximum interval allowed for this material (9 and 11 days, respectively).

Comparing both schedules (Fig. 13(a) and (b)), fewer routes are performed by vehicle 7 in the social solution (44 routes against 52 routes in the economic solution). In the social schedule, the driver of vehicle 7 works $165 \mathrm{~h}$ in collection activities, while in the economic schedule works 200 h. To reduce 35 working hours from vehicle 7 , the scheduled hours for the remaining vehicles have to increase. This can be achieved with the reconfiguration of each depot service area. As an illustrative example, the service areas for material glass for the three solutions are shown in Fig. 14. In the social solution, the number of collection sites assigned to depot 208 (114 sites) is lower than in the other two solutions (128 sites in the economic solution and 136 in the environmental solution) while the number of sites assigned to depot 209 is larger (46 sites in the social solution against 32 and 26 in the economic and environmental solutions, respectively). Depot 209 (where vehicles 5 and 6 are based) is the one with less working hours in the economic and environmental solutions (Fig. 12). In order to balance the number of working hours in the social solution, more sites have to be assigned to this depot.

The environmental solution is the one with the highest number of sites assigned to depot 208, which acts also as the sorting station. The outbound transportation is performed by larger vehicles that emit a higher value of $\mathrm{CO}_{2}$. Therefore, as the objective is to minimize the $\mathrm{CO}_{2}$ emissions, more sites are assigned to the sorting station to avoid the outbound transportation. Moreover, the environmental solution chooses routes where the vehicles travel shorter distances with heavy load given that it minimizes the $\mathrm{CO}_{2}$ emissions.

In the three solutions, a few sites are collected by inter-depot routes.

With the results of the payoff table, one determines the ranges of the objective functions that are going to be constrained in the augmented $\varepsilon$-constraint method, i.e., the environmental and social objectives. For the environmental objective range $r_{2}$, the value is $3295 \mathrm{~kg}$ of $\mathrm{CO}_{2}\left(r_{2}=z_{\max }^{2}-z_{\min }^{2}\right)$. For the social objective $r_{3}$, the value is $35 \mathrm{~h}\left(r_{3}=z_{\text {max }}^{3}-z_{\text {min }}^{3}\right)$.

After defining the ranges, it is important to define the grid size parameters $\left(q_{2}\right.$ and $\left.q_{3}\right)$. These influence the number of Paretooptimal solutions that will be found and the computational time. The total Pareto-optimal solutions can only be found if the grid is fine enough such that at most one Pareto-optimal solution is contained in each cell [57]. However, it is not our intention to generate all Pareto-optimal solutions as our ultimate goal is to identify a compromise solution and for that it is not required to generate all Pareto-optimal solutions. Only an approximation to the Pareto front is then needed and for that the use of seven grid points is sufficient $\left(q_{2}=7\right.$ and $\left.q_{3}=7\right)$. Based on this assumption, the variation of the constraints bounds to use ( $\varepsilon_{2}$ and $\left.\varepsilon_{3}\right)$ are $\Delta \varepsilon_{2}=471 \mathrm{~kg}$ of $\mathrm{CO}_{2}$ and $\Delta \varepsilon_{3}=5 \mathrm{~h}$.

Table 4 presents the 64 combinations of bounds considered to optimize the economic objective in the augmented $\varepsilon$-constraint method. All the CPLEX computational runs are limited to $1 \mathrm{~h}$. Nine 
different solutions are obtained (S1-S9 in Table 4). Such solutions can be visualized in Fig. 15, where it is shown that with improving social objective (reducing the number of maximum working hours), the economic and environmental objectives deteriorate. For instance, to improve the social objective by $17.5 \%$, the economic and the environmental objectives deteriorate $10 \%$ and $9.5 \%$, respectively (S1 versus S8). However, the economic objective only deteriorates $1.2 \%$ and the environmental $2.4 \%$ with an improvement of $12.5 \%$ in the social objective (S3 versus S8). Regarding the economic and environmental objectives, the trade-off only exists between S8 and S9. To improve $0.7 \%$ in the environmental objective, the economic objective deteriorates $0.3 \%$. In the remaining solutions, these objectives are directly proportional and in an inverse proportion to the social objective.

Aiming to find a compromise solution between the three objectives so as to plan a sustainable solution for the logistics network, the compromise solution method is applied. The ideal point is defined according to the individual minima of each objective, in this case $z_{I}=\left(27,261 \mathrm{~km}, 34,747 \mathrm{~kg} \mathrm{CO}_{2}, 165 \mathrm{~h}\right)$ and the nadir point is

Table 3

Payoff table obtained by the lexicographic optimization of the objective functions.

\begin{tabular}{lccc}
\hline & $z^{1}(\mathrm{~km})$ & $z^{2}(\mathrm{~kg})$ & $z^{3}(\mathrm{~h})$ \\
\hline $\min z^{1}$ & 27,261 & 34,982 & 200 \\
$\min z^{2}$ & 27,337 & 34,747 & 200 \\
$\min z^{3}$ & 30,118 & 38,042 & 165 \\
\hline
\end{tabular}

$z_{N}=(30,118 \mathrm{~km}, 38,042 \mathrm{~kg} \mathrm{CO}, 200 \mathrm{~h})$. After normalizing the objective functions with the amplitude between the nadir and ideal points, the compromise solution $\left(z_{C}\right)$ is obtained by minimizing the Tchebycheff distance to the ideal point. The compromise solution obtained is then $z_{C}=(28,013 \mathrm{~km}, 35,653 \mathrm{~kg} \mathrm{CO}, 174 \mathrm{~h})$. Fig. 15 also depicts the compromise solution and the ideal point.

In the compromise solution, the economic objective deteriorates $2.7 \%$, the environmental $2.6 \%$ and the social $5.5 \%$ with respect to the ideal point. This solution is detailed in Fig. 16. For all materials, the number of sites assigned to the sorting station is smaller than the ones obtained for the economic and environmental solutions, but higher than for the social solution. For instance, in the compromise solution for paper, $39 \%$ of the sites are assigned to depot 208 (sorting station), while $45 \%$ are assigned when the economic and environmental objectives are minimized individually and $38 \%$ when considering the social objective. Also more sites are collected in inter-depot routes. These differences contribute to an increase in the distance traveled and $\mathrm{CO}_{2}$ emitted but balances the solution in terms of workload among depots (Fig. 17).

The compromise solution then represents a sustainable solution that we have proposed to be implemented by the company in study. Compared to the company current solution, where the municipalities' boundaries are expected to define the service areas and the collection routes, savings of about $10 \%$ in distance and $9 \%$ in $\mathrm{CO}_{2}$ emissions and a reduction of $21 \%$ in the maximum of driving hours are obtained with the sustainable solution.
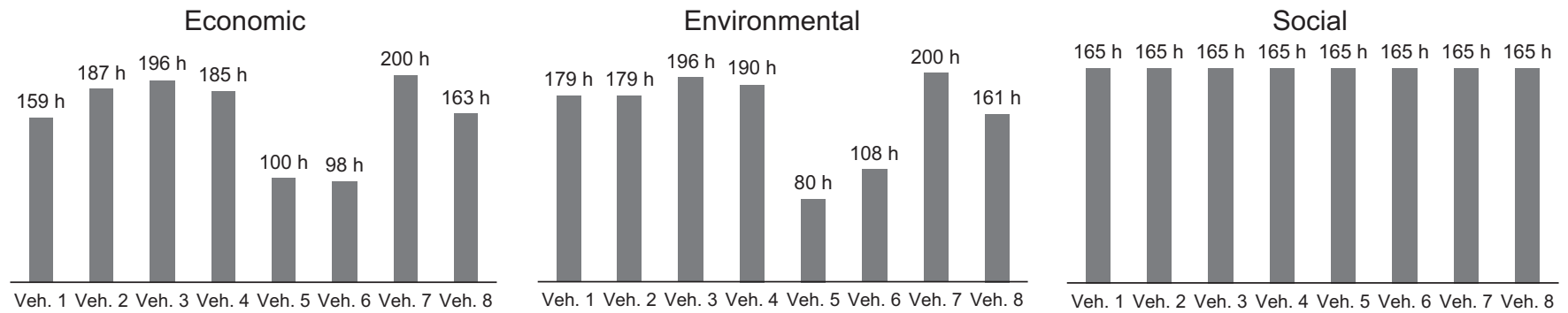

Fig. 12. Number of working hours by vehicle in the three solutions.

a

\begin{tabular}{|c|c|c|c|c|}
\hline \multicolumn{5}{|c|}{$\sum$ Hours $=200 \mathrm{~h}$} \\
\hline 1 & 2 & 3 & 4 & 5 \\
\hline \#56 (PI); & \#54 (PI); & \#253 (Pa); & \#59 (PI); & \#251 (Pa) \\
\hline \#250 (Pa) & \#249 (Pa) & \#254 (Pa) & \#63 (PI) & \\
\hline $461 \mathrm{~m}$ & $448 \mathrm{~m}$ & $480 \mathrm{~m}$ & $467 \mathrm{~m}$ & $346 \mathrm{~m}$ \\
\hline 6 & 7 & 8 & 9 & 10 \\
\hline \#61 (PI); & \#252 (Pa) & \#248 (Pa) & \#255 (Pa); & \#57 (PI); \\
\hline \#62 (PI) & & & \#267 (Pa) & \#58 (PI) \\
\hline $428 \mathrm{~m}$ & $295 \mathrm{~m}$ & $299 m$ & $387 m$ & $471 \mathrm{~m}$ \\
\hline 11 & 12 & 13 & 14 & 15 \\
\hline$\# 55(\mathrm{PI}) ; \# 60$ & \#49 (PI); & \#249 (Pa); & \#253 (Pa) & \#397 (GI) \\
\hline$(\mathrm{PI}) ; \# 81(\mathrm{Pl})$ & $\$ 250$ (Pa) & \#254 (Pa) & & \\
\hline $425 \mathrm{~m}$ & $478 \mathrm{~m}$ & $467 \mathrm{~m}$ & $295 \mathrm{~m}$ & $249 \mathrm{~m}$ \\
\hline 16 & 17 & 18 & 19 & 20 \\
\hline \#251 (Pa) & \#56 (PI); & \#54 (PI); & \#255 (Pa); & \#59 (PI); \\
\hline \#251 (Pa) & \#252 (Pa) & $\# 248(\mathrm{~Pa})$ & \#267 (Pa) & \#63 (PI) \\
\hline $346 \mathrm{~m}$ & $461 \mathrm{~m}$ & $465 \mathrm{~m}$ & $387 \mathrm{~m}$ & $467 \mathrm{~m}$ \\
\hline 21 & 22 & 23 & 24 & 25 \\
\hline \#61 (PI) & $\# 62$ (PI); & $\# 249(\mathrm{~Pa})$ & \#253 (Pa); & \#57 (PI); \\
\hline $258 m$ & $465 \mathrm{~m}$ & $282 m$ & 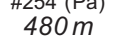 & $471 \mathrm{~m}$ \\
\hline 26 & 27 & 28 & 29 & 30 \\
\hline \#55(PI);\#60 & \#251 (Pa) & \#49 (PI); & \#248 (Pa) & \#255 (Pa); \\
\hline $\begin{array}{c}(\mathrm{PI}) ; \# 81(\mathrm{PI}) \\
425 \mathrm{~m}\end{array}$ & $346 \mathrm{~m}$ & $\begin{array}{c}\# 252(\mathrm{~Pa}) \\
478 \mathrm{~m}\end{array}$ & $299 \mathrm{~m}$ & $\begin{array}{c}\# 267(\mathrm{~Pa}) \\
387 \mathrm{~m}\end{array}$ \\
\hline
\end{tabular}

b

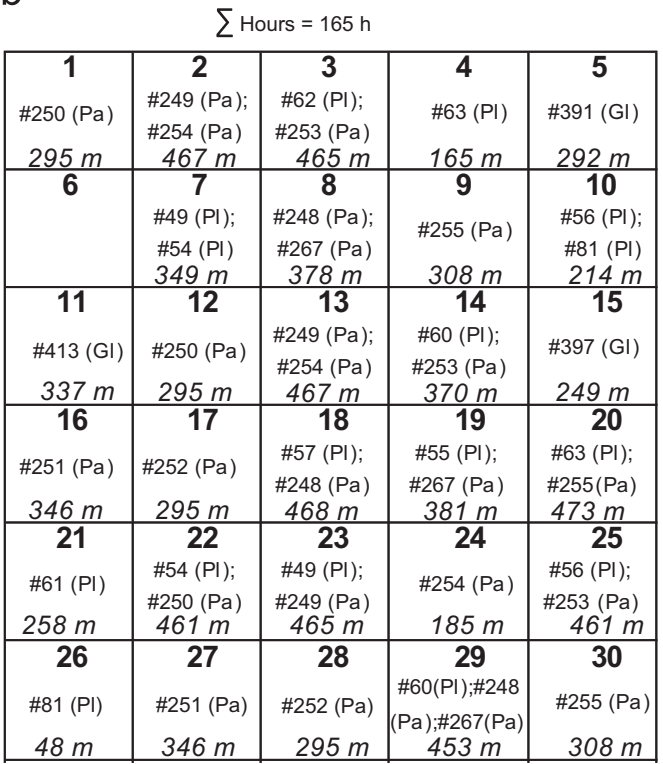

Fig. 13. Schedule for vehicle 7 in economic (a) and social (b) solutions. 


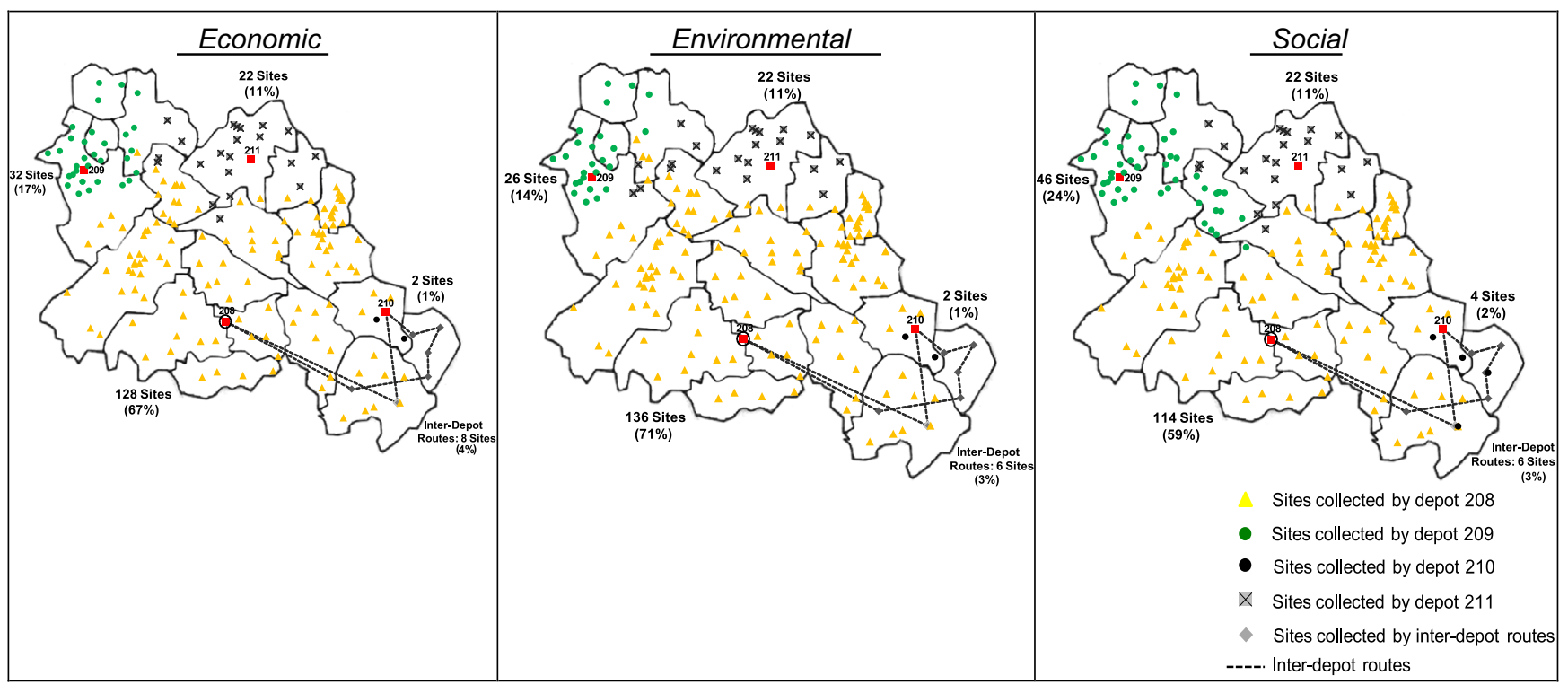

Fig. 14. Service areas for glass material for the economic, environmental and social solutions.

Table 4

Constraints bounds for the environmental $\left(\varepsilon_{2}\right)$ and social $\left(\varepsilon_{3}\right)$ objectives and Pareto-optimal solutions obtained.

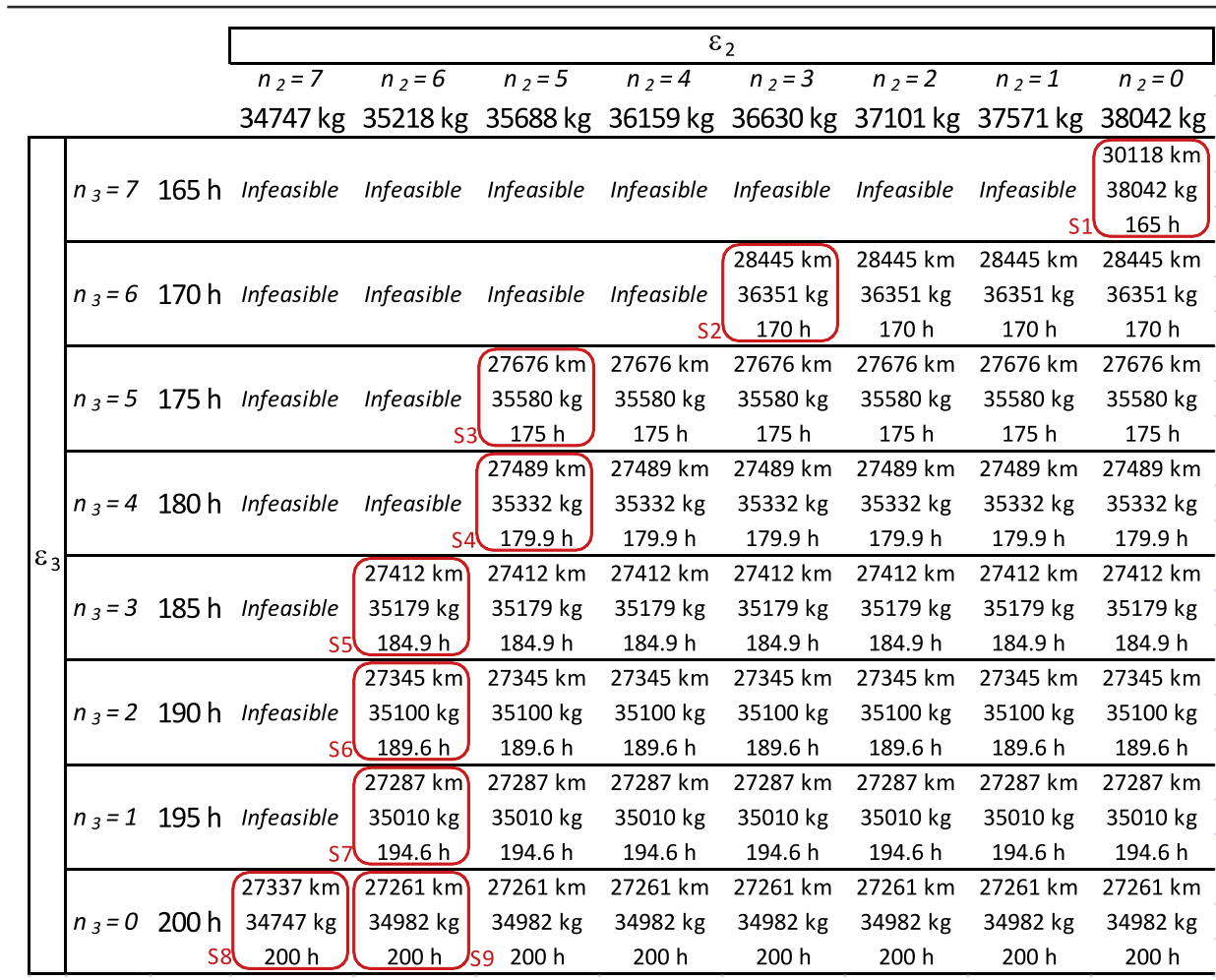

\section{Conclusions}

The problem of planning a multi-depot logistics system taking into account the three dimensions of sustainability has been studied. Economic, environmental and social objective functions are considered in a tactical routing and scheduling problem with multiple depots. The aim was to find a compromise solution between the three objectives in order to obtain a sustainable logistics system. To the best of the authors' knowledge, this problem has not yet been addressed in the existing literature.

The problem solution is achieved through the development of a multi-objective solution approach based on a set partitioning formulation. Firstly, a set of feasible routes is generated considering only the economic objective. Then, in the second step, the augmented $\varepsilon$-constraint method is applied to determine the Pareto front and the compromise solution method finds a solution 
where costs (as a linear function of the distance traveled) are balanced with environmental and social concerns. The environmental concerns are measured through the $\mathrm{CO}_{2}$ emissions of the vehicle routes, while the social concerns are measured in terms of maximum number of drivers working hours. The latter objective leads to the promotion of equity among the drivers as, in an ideal situation, all human resources should have the same number of driving hours.

The solution approach is applied to a real recyclable waste collection system, where the trade-offs between the three objectives are highlighted and a compromise solution is reached. When economic and environmental objectives are minimized, unbalanced solutions are obtained in terms of working hours by vehicle. On the contrary, when the social objective is minimized, a balanced solution is obtained, where all drivers have to drive the same number of hours. However, this equity solution leads to a significant increase in distance and $\mathrm{CO}_{2}$ emissions. Between environmental and economic objectives there are only slight trade-offs, indicating that the threeobjective model could collapse into a two-objective model. An efficient solution taking into account the three objectives is obtained through the compromise solution method, where the distance to the ideal point is minimized.

As the main conclusion it can be stated that an innovative approach to planning sustainable logistics system has been

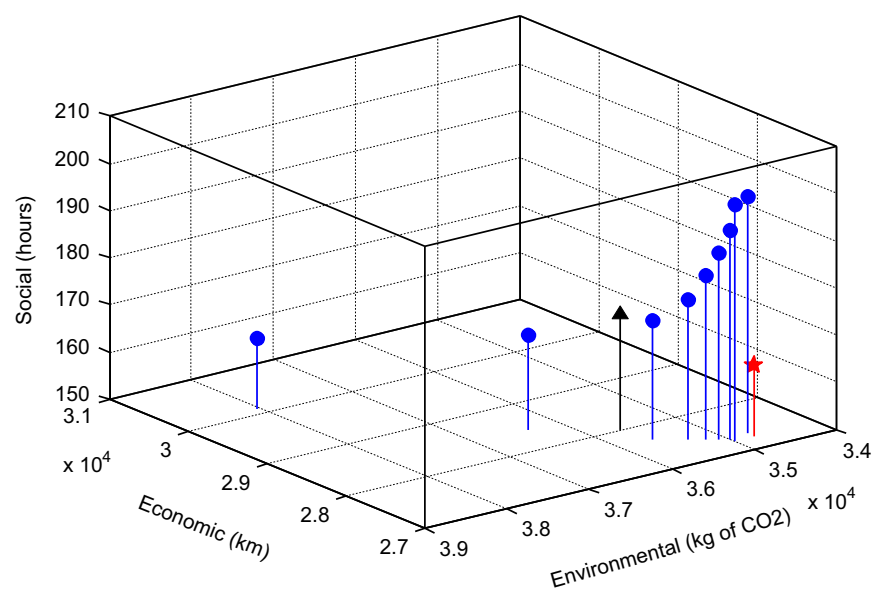

- Pareto optimal solutios
- Compromise solution

$\star$ Ideal solution
A Compromise

Fig. 15. Approximation to Pareto front considering the three objectives with the ideal point and the compromise solution highlighted. proposed. Tactical and operational decisions are considered simultaneously and different solutions are obtained when each dimension of sustainability is addressed individually. The main contribution of this work is to integrate within a single solution the three dimensions of sustainability, where new aspects that should be considered when planning reverse logistic systems are modeled. In particular, service areas, routes definition as well as routes scheduling, $\mathrm{CO}_{2}$ emissions and human resources working hours have been considered.

As future work, a sensitivity analysis to some model's parameters could be performed in order to assess how the Pareto front reacts to changes in some parameters. Also further investment should be made on measures for economic and social objectives. On the economic side, a common assumption of routing models (and used in this work) is that the variable costs depend linearly on the distance traveled, leading to an objective function that minimizes distance. However, this may be improved and a more comprehensive function could be developed to measure variable costs. For instance, fuel consumption and vehicle maintenance could be also modeled. The former depends on distance, load, speed and vehicle features, and the latter depends on distance traveled and vehicles typology. On the social objective, other measures to account for such objective should be investigated. The social objective is the less studied of the three sustainability dimensions and further work should then be performed in this area. Moreover, this work can be extended to cope with strategic decisions like depots location and/or vehicle fleet sizing and location in order to promote the logistics system's sustainability as a whole.

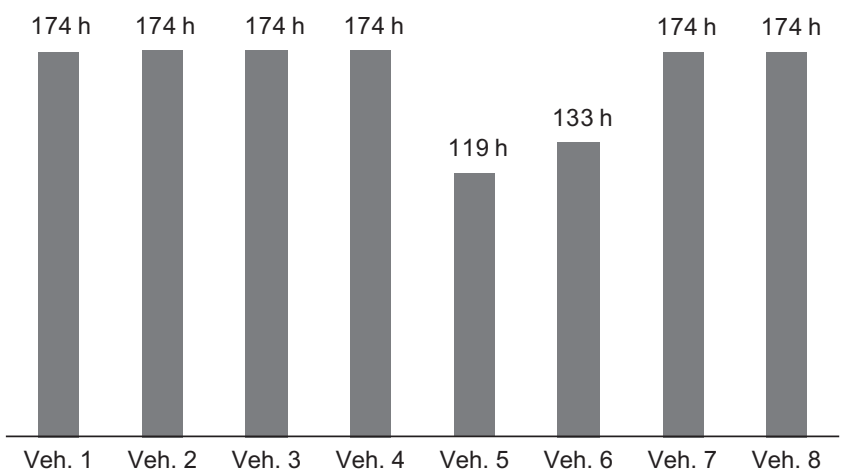

Fig. 17. Number of working hours by vehicle in the compromise solution.

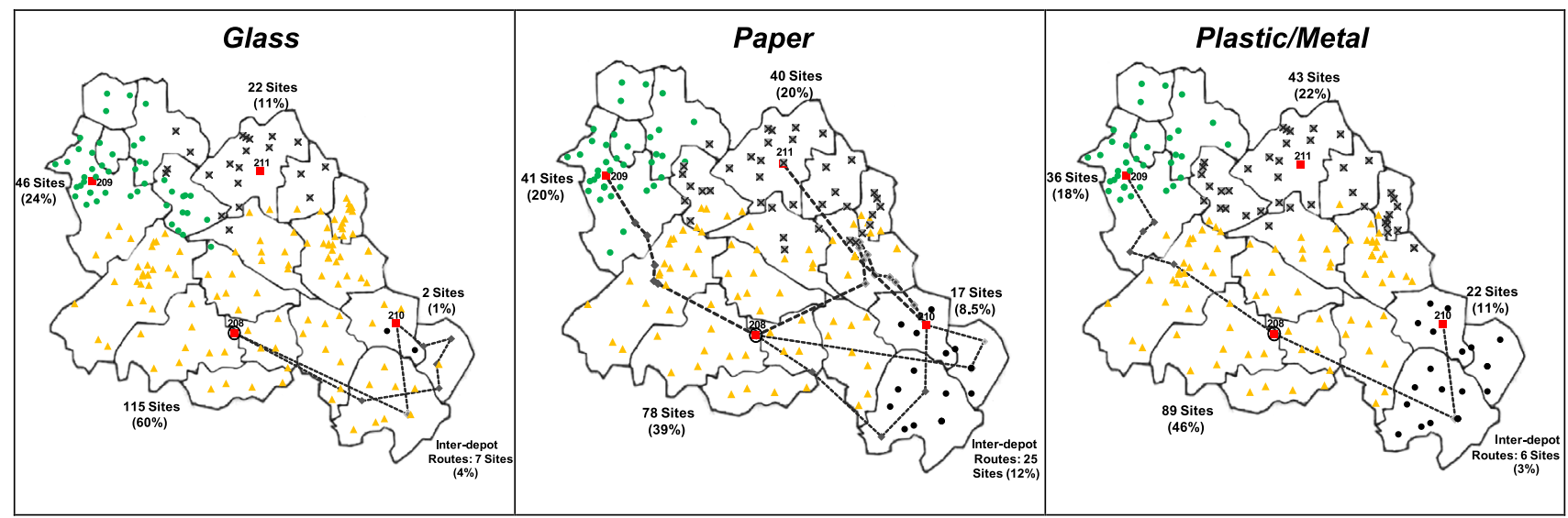

Fig. 16. Representation of the compromise solution for the three recyclable materials. 


\section{Acknowledgment}

The insightful contributions of the two anonymous referees are gratefully acknowledged.

\section{References}

[1] World Commission on Environment and Development. Our common future. Oxford, UK: Oxford University Press; 1987.

[2] Elkington J. Cannibals with forks: the triple bottom line of the 21st century. London, UK: Earthscan; 1997.

[3] Sbihi A, Eglese RW. Combinatorial optimization and green logistics. 40R-A Quarterly Journal of Operations Research 2007;5:99-116.

[4] Tang CS, Zhou S. Research advances in environmentally and socially sustainable operations. European Journal of Operational Research 2012;223:585-594.

[5] Frota JQ, Bloemhof-Ruwaard JM, van Nunen J, van Heck E. Designing and evaluating sustainable logistics networks. International Journal of Production Economics 2008;111:195-208.

[6] Dekker R, Bloemhof J, Mallidis I. Operations Research for green logistics-an overview of aspects, issues, contributions and challenges. European Journal of Operational Research 2012;219:671-679.

[7] Bektas T, Laporte G. The pollution-routing problem. Transportation Research Part B Methodological 2011;45:1232-1250.

[8] Ubeda S, Arcelus FJ, Faulin J. Green logistics at Eroski: a case study. International Journal of Production Economics 2011;131:44-51.

[9] Erdogan S, Miller-Hooks E. A Green Vehicle Routing Problem. Transportation Research Part E: Logistics and Transportation Review 2012;48:100-114.

[10] Sheu JB, Chou YH, Hu CC. An integrated logistics operational model for greensupply chain management. Transportation Research Part E: Logistics and Transportation Review 2005;41:287-313.

[11] Gu QL, Ji JH. An integrated logistics operational model for remanufacturing/ manufacturing system based on the consumer market. International Journal of Logistics Systems and Management 2008;4:21-29.

[12] Srivastava SK. Network design for reverse logistics. Omega-International Journal of Management Science 2008;36:535-548.

[13] Lee DH, Dong M, Bian W. The design of sustainable logistics network under uncertainty. International Journal of Production Economics 2010;128: 159-166.

[14] Salema MIG, Barbosa-Povoa AP, Novais AQ. A strategic and tactical model for closed-loop supply chains. OR Spektrum 2009:31:573-599.

[15] Salema MIG, Barbosa-Povoa AP, Novais AQ. Simultaneous design and planning of supply chains with reverse flows: a generic modelling framework. European Journal of Operational Research 2010;203:336-349.

[16] Qiang Q, Ke K, Anderson T, Dung J. The closed-loop supply chain network with competition, distribution channel investment, and uncertainties. OmegaInternational Journal of Management Science 2013;41:186-194.

[17] Labuschagne C, Brent AC, van Erck RPG. Assessing the sustainability performances of industries. Journal of Cleaner Production 2005;13:373-385.

[18] Ramos TRP, Oliveira RC. Delimitation of service areas in reverse logistics networks with multiple depots. Journal of Operational Research Society 2011;62:1198-1210.

[19] Faulin J, Lera-Lopéz F, Juan A. Optimizing routes with safety and environmental criteria in transportation management in Spain: a case study. International Journal of Information Systems and Supply Chain Management 2011;4:38-59.

[20] Li JQ Borenstein D, Mirchandania PB. Truck scheduling for solid waste collection in the City of Porto Alegre, Brazil. Omega-International Journal of Management Science 2008;36:1133-1149.

[21] Pasia JM, Doerner KF, Hartl RF, Reimann M. Solving a bi-objective vehicle routing problem by pareto-ant colony optimization. In: Stutzle T, Birattari M, Hoos $\mathrm{HH}$, editors. Engineering stochastic local search algorithms: designing, implementing and analyzing effective heuristics. Berlin: Springer-Verlag; 2007. p. $187-191$.

[22] Pasia JM, Doerner KF, Hartl RF, Reimann M. A population-based local search for solving a bi-objective vehicle routing problem. In: Cotta C, VanHemert J, editors. Proceedings of evolutionary computation in combinatorial optimization. Berlin: Springer-Verlag; 2007. p. 166-175.

[23] Jozefowiez N, Semet F, Talbi EG. Target aiming Pareto search and its application to the vehicle routing problem with route balancing. Journal of Heuristics 2007; 13:455-469.

[24] Jozefowiez N, Semet F, Talbi EG. An evolutionary algorithm for the vehicle routing problem with route balancing. European Journal of Operational Research 2009;195:761-769.

[25] Reiter P, Gutjahr WJ. Exact hybrid algorithms for solving a bi-objective vehicle routing problem. Central European Journal of Operational Research 2012;20:19-43.

[26] Garetti M, Taisch M. Sustainable manufacturing: trends and research challenges. Production Planning \& Control 2012;23:83-104.

[27] Petch RJ, Salhi S. A multi-phase constructive heuristic for the vehicle routing problem with multiple trips. Discrete Applied Mathematics 2003;133:69-92.
[28] Olivera A, Viera O. Adaptive memory programming for the vehicle routing problem with multiple trips. Computers \& Operations Research 2007;34: $28-47$.

[29] Rieck J, Zimmermann J. A new mixed integer linear model for a rich vehicle routing problem with docking constraints. Annals of Operations Research 2010;181:337-358.

[30] Azi N, Gendreau M, Potvin JY. An exact algorithm for a vehicle routing problem with time windows and multiple use of vehicles. European Journal of Operational Research 2010;202:756-763.

[31] Tan CCR, Beasley JE. A heuristic algorithm for the period vehicle-routing problem. Omega-International Journal of Management Science 1984;12: 497-504.

[32] Tung DV, Pinnoi A. Vehicle routing-scheduling for waste collection in Hanoi. European Journal of Operational Research 2000;125:449-468.

[33] Angelelli E, Speranza MG. The periodic vehicle routing problem with intermediate facilities. European Journal of Operational Research 2002;137: 233-247.

[34] Renaud J, Laporte G, Boctor FF. A tabu search heuristic for the multi-depot vehicle routing problem. Computers \& Operations Research 1996;23:229-235.

[35] Cordeau JF, Gendreau M, Laporte G. A tabu search heuristic for periodic and multi-depot vehicle routing problems. Networks 1997;30:105-119.

[36] Hadjiconstantinou E, Baldacci R. A multi-depot period vehicle routing problem arising in the utilities sector. Journal of Operational Research Society 1998;49: 1239-1248.

[37] Parthanadee P, Logendran R. Periodic product distribution from multi-depots under limited supplies. IIE Transactions 2006;38:1009-1026.

[38] Vidal T, Crainic TG, Gendreau M, Lahrichi N, Rei W. A hybrid genetic algorithm for multidepot and periodic vehicle routing problems. Operations Research 2012;60:611-624.

[39] Filipec M, Skrlec D, Krajcar S. Genetic algorithm approach for multiple depot capacitated vehicle routing problem solving with heuristic improvements built-in. International Journal of Modelling and Simulation 2000;20:320-328.

[40] Ramos TRP, Gomes MI, Barbosa-Povoa AP. Planning waste cooking oil collection systems. Waste Management 2013;33:1691-1703.

[41] Crevier B, Cordeau JF, Laporte G. The multi-depot vehicle routing problem with inter-depot routes. European Journal of Operational Research 2007;176: 756-773.

[42] Tarantilis CD, Zachariadis EE, Kiranoudis CT. A hybrid guided local search for the vehicle-routing problem with intermediate replenishment facilities. Informs Journal on Computing 2008;20:154-168.

[43] Ramos TRP. Tactical and operational planning in reverse logistics systems with multiple depots. Instituto Superior Técnico, Universidade Técnica de Lisboa; 2012 ([Ph.D. thesis]).

[44] Laporte G. What you should know about the vehicle routing problem. Naval Research Logistics 2007;54:811-819.

[45] Baldacci R, Hadjiconstantinou E, Mingozzi A. An exact algorithm for the capacitated vehicle routing problem based on a two-commodity network flow formulation. Operations Research 2004;52:723-738.

[46] Barth M, Scora G, Younglove T. Modal emissions model for heavy-duty diesel vehicles. Transportation Research Record 2004;1880:10-20.

[47] Department for Environment, Food and Rural Affairs (Defra). Guidelines to Defra/DECC's GHG conversion factors for company reporting; 2012 〈http:// www.defra.gov.uk/environment/economy/business-efficiency/reporting, accessed 21.03〉.

[48] Coello CAC, Romero CEM. Evolutionary algorithms and multiple objective optimization. In: M. Ehrgott, X. Gandibleux, editors, Multiple criteria optimization: state of the art annotated bibliographic surveys; 2003. p. 277-331.

[49] Hwang CL, Masud AS. Multiple objective decision making, methods and applications: a state-of-the-art survey; 1979.

[50] Berube JF, Gendreau M, Potvin JY. An exact epsilon-constraint method for biobjective combinatorial optimization problems: application to the traveling salesman problem with profits. European Journal of Operational Research 2009;194:39-50.

[51] Grandinetti L, Guerriero F, Lagana D, Pisacane O. An optimization-based heuristic for the multi-objective undirected capacitated arc routing problem. Computers \& Operations Research 2012;39:2300-2309.

[52] Liu S, Papageorgiou LG. Multiobjective optimisation of production, distribution and capacity planning of global supply chains in the process industry. Omega-International Journal of Management Science 2013;41:369-382.

[53] Current J, Marsh M. Multiobjective transportation network design and routing-problems taxonomy and annotation. European Journal of Operational Research 1993;65:4-19.

[54] Ehrgott M, Gandibleux X. A survey and annotated bibliography of multiobjective combinatorial optimization. OR Spektrum 2000;22:425-460.

[55] Mavrotas G. Effective implementation of the epsilon-constraint method in multi-objective mathematical programming problems. Applied Mathematics and Computation 2009;213:455-465.

[56] Yu PL. Multiple criteria decision making: concepts, techniques and extensions New York: Plenum Press; 1985.

[57] Laumanns M, Thiele L, Zitzler E. An efficient, adaptive parameter variation scheme for metaheuristics based on the epsilon-constraint method. European Journal of Operational Research 2006;169:932-942. 